\title{
User Association in Energy-Aware Dense Heterogeneous Cellular Networks
}

DOI:

10.1109/TWC.2017.2653107

\section{Document Version}

Accepted author manuscript

Link to publication record in Manchester Research Explorer

\section{Citation for published version (APA):}

Mugume, E., \& So, K. C. (2017). User Association in Energy-Aware Dense Heterogeneous Cellular Networks. IEEE Transactions on Wireless Communications, 16(3). https://doi.org/10.1109/TWC.2017.2653107

\section{Published in:}

IEEE Transactions on Wireless Communications

\section{Citing this paper}

Please note that where the full-text provided on Manchester Research Explorer is the Author Accepted Manuscript or Proof version this may differ from the final Published version. If citing, it is advised that you check and use the publisher's definitive version.

\section{General rights}

Copyright and moral rights for the publications made accessible in the Research Explorer are retained by the authors and/or other copyright owners and it is a condition of accessing publications that users recognise and abide by the legal requirements associated with these rights.

\section{Takedown policy}

If you believe that this document breaches copyright please refer to the University of Manchester's Takedown Procedures [http://man.ac.uk/04Y6Bo] or contact uml.scholarlycommunications@manchester.ac.uk providing relevant details, so we can investigate your claim.

\section{OPEN ACCESS}




\title{
User Association in Energy-Aware Dense Heterogeneous Cellular Networks
}

\author{
Edwin Mugume, Member, IEEE, and Daniel K. C. So, Senior Member, IEEE
}

\begin{abstract}
Mobile traffic demand has been increasing exponentially over the last few years and forecasts show that this trend will continue in the foreseeable future. As a result, operators are forced to densify and upgrade their networks to meet this demand. This has created concerns such as increasing greenhouse gas emissions, high capital expenditures and associated energy costs. This paper uses tools from stochastic geometry to analyze and formulate energy-efficient deployment strategies for multi-tier heterogeneous networks (HetNets) using various user association schemes. We use simple approximations to combine the required base station (BS) density and associated transmit power per tier subject to both coverage probability and average user rate constraints. In this paper, this combination is called the deployment factor and it can be expressed in closed form for unbiased HetNets. We then formulate an area power consumption (APC) minimization framework which optimizes the deployment factor to derive specific optimal BS density and transmit power values. Furthermore, we perform a comprehensive study of the effect of biasing on the APC performance of biased HetNets. Our results show that for HetNets using the maximum average-biased-received-power (ABRP) association scheme, significant energy savings are possible with appropriate biasing.
\end{abstract}

\section{Index Terms}

Heterogeneous networks, user association, coverage probability, average user rate, deployment configuration, biasing, area power consumption.

\section{INTRODUCTION}

Mobile network operators are currently faced with exponentially increasing data demand as a result of significant improvement in cellular access technologies and the development of smart

The authors are with the Microwave and Communication Systems Group, School of Electrical and Electronic Engineering, The University of Manchester, Manchester, M13 9PL, United Kingdom. Email: edwin.mugume@gmail.com, d.so@manchester.ac.uk. 
devices and their data-hungry applications [1], [2]. A Cisco forecast has predicted that this trend will continue in the future with a tenfold increase from 2014 to 2019 [1]. This is a big challenge for operators to meet this demand because the usable spectrum is already congested and expensive yet radio links are close to their theoretical capacity limit. Researchers in both academia and industry are actively designing next-generation technologies that will enable operators to continue providing reliable quality of service to their subscribers. One solution that has shown great promise is the densification of existing networks with more base stations (BSs) to enhance frequency reuse and therefore increase the average bandwidth per user [3]. However, network densification also increases both capital and operational expenses, particularly the cost of energy, and the associated greenhouse gas emissions that enhance global warming. Other techniques to increase network capacity include MIMO and massive MIMO systems [4], [5], cognitive radio systems [6], etc. In addition, future 5G systems are expected to provide up to 1000 times more area spectral efficiency compared to current 4G technology [7], [8].

\section{A. Related Work}

Motivated by these challenges, researchers have proposed a new cellular architecture called a heterogeneous cellular network (HetNet) in which different types of BSs are deployed in a multi-tier hierarchical structure to improve the spectral efficiency (SE) of the network [9], [10]. A HetNet essentially combines macro BSs that cover relatively large areas with tiers of low-power and small coverage BSs such as micro BSs, pico BSs and femto BSs. Small BSs are mainly used to densify an existing macrocell network by providing targeted coverage and capacity enhancement in dense urban and suburb areas [11]. Small BSs are used to offload traffic from congesting macro BSs so as to achieve load balancing and enhance quality of service. However, they are generally dominated by macro BSs which transmit at significantly higher power levels. Therefore, to enhance load balancing, a simple technique called biasing is used to artificially bias user association to the small cell tiers. Also called range extension, each tier is assigned with a bias value (depending on its transmit power and/or BS density) which makes small cell tiers relatively more attractive to users than the macrocell tier [10], [13], [14].

Cellular networks are traditionally planned to support peak traffic demand. However, studies have shown that traffic demand varies significantly in both time and space domains [12]. During low traffic periods, many small BSs in a dense HetNet may remain underutilized or idle which can have a negative impact on the energy performance of the HetNet. Several works have discussed 
and tried to address this issue by proposing sleep mode mechanisms whose main purpose is to adapt the energy consumption of the HetNet with changes in traffic demand [15]-[17]. For example, the density of active BSs may be reduced or idle BSs may put to sleep mode when traffic is low. Furthermore, jointly optimizing the SE and energy efficiency (EE) of the HetNet can exploit their inherent tradeoffs to improve overall bandwidth and energy utilization [18].

From an energy consumption perspective, the combination of BS densities and their associated transmit powers per tier in a multi-tier HetNet is an important optimization problem [19], [20]. The authors in [19] design an energy cost minimization framework to determine the optimal BS densities per tier in a two-tier unbiased HetNet subject to a service outage constraint. Service outage occurs when the instantaneous downlink rate of the typical user falls below a threshold. Although transmit power significantly influences the energy consumption of each BS, this work ignores its optimization. In addition, it is assumed that the HetNet is unbiased and a coverage probability constraint is not considered in the optimization.

An improvement of this work is shown in [20] where the authors determine the deployment factor of the HetNet, which is basically an expression that combines the BS densities and transmit powers of all tiers in some mathematical form. In [20], the optimal deployment factor of a two-tier unbiased HetNet is expressed as $A^{\star}=\lambda_{M} P_{M}^{2 / \alpha}+\lambda_{m} P_{m}^{2 / \alpha}$, where $\lambda_{M}$ and $\lambda_{m}$ are the macro BS and micro BS densities while $P_{M}$ and $P_{m}$ are their respective transmit powers. The authors then develop an area power consumption (APC) minimization framework that optimizes the deployment factor to obtain the optimal BS density and transmit power per tier subject to a coverage probability constraint. However, this work ignores an average rate constraint and also assumes that the HetNet is unbiased. Moreover, $A^{\star}$ is not expressed in closed form and can only be determined using numerical methods.

\section{B. Contributions and Organization}

In this paper, we use the stochastic geometry approach to investigate energy consumption aspects of general multi-tier HetNets. In particular, we analyze user association schemes to minimize the APC of the network under rate and coverage constraints. APC is chosen as the objective function because in HetNets, there can be many BSs in a given area consuming a large aggregate amount of power. While conventional EE optimization can minimize the overall EE of the network, it cannot guarantee the minimization of power consumption which is a key parameter for network operators. Our constrained optimization framework using APC as the 
objective function ensures that the overall power consumption is minimized while maintaining acceptable network performance in terms of coverage and rate.

Stochastic geometry has emerged as a popular approach to perform simple and tractable analysis that describes various performance aspects of homogeneous networks [15], [17], [21][23] and HetNets [19], [20], [24]-[27]. Since HetNets combine BSs of different types, they are characterized by highly varying cell sizes and shapes. Using the Poisson Point Process (PPP) to model the locations of BSs in each tier uniquely simulates this variability of cell sizes and shapes. Moreover, this methodology avoids relying on Monte Carlo simulations to evaluate performance because they are very intensive and results are difficult to verify.

The work in this paper relies on some of the analysis of the coverage probability and average rate performance of a HetNet derived in [14], [15] and [24]. Although the impact of scheduling on resource allocation is beyond the scope of our analysis, later works such as [13], [27] have discussed its negative effect on the resulting network capacity. This paper also builds on our previous work in [28] which considers a HetNet using only one user association scheme. In general, our paper makes the following major contributions:

- Using tools from stochastic geometry, we manipulate the expressions of coverage probability and average rate to formulate a constrained deployment strategy that minimizes the energy consumption of a HetNet based on three different user association schemes. This deployment strategy is expressed in terms of the deployment factor of the HetNet, similar to [20].

- Using appropriate and verifiable approximations, we express the deployment factor of the unbiased HetNet in closed form for two of the user association schemes. This analysis is extended to a biased two-tier HetNet whose deployment factor is determined numerically subject to reasonable network deployment assumptions.

- Given the deployment factor for both biased and unbiased HetNet based on the three user association schemes, we formulate a theoretical optimization framework to determine the optimal combination of BS densities and associated transmit powers per tier that minimize the APC of the HetNet subject to coverage and rate constraints.

- We justify that both coverage and average rate constraints are essential to achieving performance targets of both measures in all cellular environments. In addition, we comprehensively investigate the effect of biasing on the APC of the HetNet under realistic network conditions. Therefore, our joint optimization of the BS densities and associated transmit powers subject to coverage and average rate constraints exceeds existing strategies which consider either: (i) 
optimization of the BS density only [19]; (ii) only an unbiased HetNet subject to only the coverage constraint [20]; or (iii) only one user association scheme [19], [20], [28].

The rest of this paper is organized as follows. Section II presents the network topology, user association schemes and BS power consumption models. Section III presents performance analysis of the HetNet using any of three user association schemes. Section IV presents the optimal HetNet deployment strategy that minimizes the APC. Section V presents and discusses the numerical results. The paper is concluded in Section VI.

\section{System MODEL}

\section{A. Network Topology}

Consider a general $K$-tier HetNet consisting of a tier of macro BSs and $(K-1)$ tiers of small BSs, all independently located on the 2-D Euclidean plane. BS locations in the $k$-th tier are modeled according to a homogeneous PPP $\Phi_{k}$ of density $\lambda_{k}$. In addition, each $k$-th tier BS transmits the same power $P_{k}$ and is assigned an association bias value of $\beta_{k}$. The association bias $\beta_{k}$ describes the degree to which a typical user is artificially manipulated to favour connecting to the $k$-th tier BSs relative to the BSs in other tiers. Hence each tier is uniquely described by the tuple $\left(\lambda_{k}, P_{k}, \beta_{k}\right)$. Shadowing is ignored such that the HetNet layout resembles the weighted Poisson-Voronoi tessellation [19].

Without loss of generality, consider a typical user located at the origin and at a distance $r_{k}$ from its serving $k$-th tier BS. The typical user is defined as a randomly selected user in the PPP-based HetNet based on Slivnyak's theorem, which states that the distribution of the original PPP is equivalent to its reduced Palm distribution. In other words, the properties of the PPP are invariant to translation [21]. The considered pathloss model is $l\left(r_{k}\right)=L\left\|r_{k}\right\|^{-\alpha}$ where $L$ is a pathloss constant and $\alpha>2$ is the pathloss exponent. The fading loss is assumed to be i.i.d exponential i.e. $h_{r_{k}} \sim \exp (1)$. Therefore, the power received by the typical user from its serving BS is $P_{r, k}=P_{k} L h_{r_{k}}\left\|r_{k}\right\|^{-\alpha}$. Universal frequency reuse is considered such that a typical user receives interference from every active BS other than its parent BS. The resulting SINR is therefore expressed as

$$
\operatorname{SINR}\left(r_{k}\right)=\frac{P_{k} h_{r_{k}}\left\|r_{k}\right\|^{-\alpha}}{\frac{\sigma^{2}}{L}+I_{j}},
$$

where interference $I_{j}=\sum_{j=1}^{K} \sum_{r_{j} \in \Phi_{j} \backslash r_{k}} P_{j} h_{r_{j}}\left\|r_{j}\right\|^{-\alpha}$ and $\sigma^{2}$ is the additive noise power. 


\section{B. User Association Schemes}

We consider the coverage probability and average rate performance of the HetNet under three user association schemes namely; (i) Maximum average biased received power (ABRP) scheme [14]; (ii) Minimum biased transmission distance (BTD) scheme [15]; and (iii) Maximum instantaneous SINR (i-SINR) scheme [24].

In maximum ABRP connectivity, a user connects to the BS that provides the strongest average biased received power i.e. the user connects to the nearest $k$-th tier $\mathrm{BS}$ if $P_{r, k}>P_{r, j}$ for all $j \in K, j \neq k$, where the long-term average biased received power $P_{r, k}=P_{k} \beta_{k} l\left(r_{k}\right)$. Therefore, a user is more likely to associate to a tier with a larger BS density, transmit power and bias factor [14]. In maximum i-SINR connectivity, a user connects to the BS that provides the highest instantaneous SINR [24]. In minimum BTD connectivity, it is assumed that a user knows its relative distances to each of its neighboring BSs in all tiers. If the nearest $k$-th tier BS is located at a distance $r_{k}$ from the user, then $r_{k}$ is multiplied by its respective bias factor $v_{k}$ [15]. The user then associates to the $k$-th tier if $v_{k} r_{k}<v_{j} r_{j}$ for all $j \in K, j \neq k$.

\section{BS Power Model}

The total amount of power consumed by a BS depends on its type and operating mode. Macro BSs generally consume more power than small BSs such as micro and pico BSs. In addition, a BS consumes the most power in active mode which includes components such as the power amplifier, signal processing, power supply losses, cooling, antenna and feeder losses, etc. When a BS is in idle mode, it still consumes a significant but fixed amount of power. In sleep mode, the BS switches off most components and the power consumption significantly reduces. In general, the power consumption of a $k$-th tier BS in active mode is expressed as [29]

$$
P_{\text {cons }, k}=N_{k} P_{0, k}+\Delta_{k} P_{k}
$$

where $N_{k}$ is the number of transceiver chains, $P_{0, k}$ is the fixed power consumption at zero load, $\Delta_{k}$ is the slope of the load-dependent power consumption and $P_{k} \in\left[0, \bar{P}_{k}\right]$ where $\bar{P}_{k}$ is the maximum transmit power level. In this paper, the APC is used to quantify the energy performance of the HetNet. In a general $K$-tier HetNet, the APC (in Watts $/ \mathrm{m}^{2}$ ) is expressed as

$$
\mathrm{APC}=\sum_{k=1}^{K} \lambda_{k}\left(N_{k} P_{0, k}+\Delta_{k} P_{k}\right) .
$$

We refer to [29] to quantify the power consumption of various BS types. 


\section{HetNet Performance AnAlysis}

To optimize the deployment configuration of a HetNet, we consider both coverage probability and average user rate constraints to guarantee that performance targets in both measures are achieved in all network environments. As the BS density in the HetNet increases, aggregate interference also increases and the overall effect of noise on coverage probability and average rate performance reduces. In other words, the interference-limited HetNet (a scenario where $\sigma^{2}=0$ ) defines the upper bound of both performance measures [24].

Biasing in HetNets has important performance benefits such as load balancing which may enhance the overall HetNet performance. However, artificial biasing is known to reduce coverage probability and spectral efficiency (in $\mathrm{b} / \mathrm{s} / \mathrm{Hz}$ ) of the HetNet since some users are forced to connect to less favorable BSs [14]. To facilitate its application in the HetNet, each performance target is set as a fraction of its respective upper bound level. In other words, coverage probability and average user rate constraints are expressed in terms of their upper bound values as

$$
\mathcal{P}_{c}=\epsilon \overline{\mathcal{P}}_{c} \text { and } \mathcal{R}_{u}=\kappa \overline{\mathcal{R}}_{u}
$$

respectively, where $\mathcal{P}_{c}$ and $\mathcal{R}_{u}$ are the coverage probability and average user rate of the HetNet, $\overline{\mathcal{P}}_{c}$ and $\overline{\mathcal{R}}_{u}$ represent their upper bound values, and $\epsilon \in(0,1]$ and $\kappa \in(0,1]$ are the ratios of coverage probability and average user rate to their respective upper bound values.

It is assumed that all tiers have the same pathloss exponent $\alpha$. This assumption facilitates important approximations of coverage probability and average user rate which are applied in HetNet optimization to devise its optimal deployment factor [20]. In addition, the analysis of minimum BTD scheme relies on this assumption to simplify the Laplace transform of its aggregate interference and express its analytical performance in a form that is easily and directly comparable to both maximum ABRP and maximum i-SINR association schemes.

The coverage probability and average rate experienced by a given user depends on the deployment configuration $\left(\lambda_{k}, P_{k}, \beta_{k}\right)$ of the tier it is associated to and the SINR threshold, denoted as $T$. For a user connected to the $k$-th tier, denote the $k$-th tier coverage probability and average user rate as $\mathcal{P}_{c, k}$ and $\mathcal{R}_{u, k}$ respectively. These values are then used to obtain the overall average HetNet performance using the law of total probability as [14]

$$
\mathcal{P}_{c}=\sum_{k=1}^{K} \mathcal{P}_{c, k} \mathcal{A}_{k} \text { and } \mathcal{R}_{u}=\sum_{k=1}^{K} \mathcal{R}_{u, k} \mathcal{A}_{k}
$$

where $\mathcal{A}_{k}$ is the $k$-th tier association probability. 
Consider a typical user located at the origin and assume that it is associated to a $k$-th tier $\mathrm{BS}$ located at a distance $r_{k}$ from the origin. The coverage probability of the $k$-th tier is then expressed as [14], [24]

$$
\begin{aligned}
\mathcal{P}_{c, k} & =\mathbb{E}_{x}\left[\mathbb{P}\left(\operatorname{SINR}_{k}(x)>T\right)\right] \\
& =\int_{0}^{\infty} e^{-\left(\frac{T \sigma^{2}}{P_{k} L} x^{\alpha}\right)} \prod_{j=1}^{K} \mathcal{L}_{I_{j}}\left(s_{c}\right) f_{X_{k}}(x) \mathrm{d} x,
\end{aligned}
$$

where $\mathcal{L}_{I_{j}}\left(s_{c}\right)$ is the Laplace transform of interference evaluated at $s_{c}=T x^{\alpha} P_{k}^{-1}$ and $f_{X_{k}}(x)$ is the PDF of the distance between the user and serving BS and depends on the association scheme. Similarly, the average rate of a user associated to the $k$-th tier is expressed as [14], [24]

$$
\begin{aligned}
\mathcal{R}_{u, k} & =\int_{x>0} \int_{t>0} \mathbb{P}\left(\log _{2}\left(1+\operatorname{SINR}_{k}(x)\right)>t\right) f_{X_{k}}(x) \mathrm{d} t \mathrm{~d} x \\
& =\Xi\left[e^{-\left(\frac{\sigma^{2}}{P_{k} L}\left(2^{t}-1\right) x^{\alpha}\right)} \prod_{j=1}^{K} \mathcal{L}_{I_{j}}\left(s_{r}\right) f_{X_{k}}(x)\right]
\end{aligned}
$$

where $\Xi[f(x, t)]=\int_{t>0} \int_{x>0} f(x, t) \mathrm{d} x \mathrm{~d} t, t$ is a random variable and $\mathcal{L}_{I_{j}}\left(s_{r}\right)$ is evaluated at $s_{r}=x^{\alpha} P_{k}^{-1}\left(2^{t}-1\right)$.

The fundamental coverage probability and average user rate analysis of a HetNet using maximum ABRP and maximum i-SINR association schemes is discussed in [14] and [24] respectively. For completeness, these results are summarized next. The work in this paper builds on this analysis to perform a constrained optimization of the deployment configuration of the HetNet using these association schemes.

The coverage probability of a typical user in a HetNet using maximum ABRP connectivity is expressed as [14]

$$
\mathcal{P}_{c_{P}}=\sum_{k=1}^{K} \pi \lambda_{k} \int_{z>0} e^{-\frac{T \sigma^{2}}{P_{k} L} z^{\alpha / 2}} e^{-\pi \sum_{j=1}^{K} \lambda_{j} \widehat{P}_{j}^{2 / \alpha} \mathcal{C}_{j} z} \mathrm{~d} z
$$

where $\widehat{P}_{j}=\frac{P_{j}}{P_{k}}, \mathcal{C}_{j}=\widehat{\beta}_{j}^{2 / \alpha}+\mathcal{Z}\left(T, \alpha, \widehat{\beta}_{j}\right), \widehat{\beta}_{j}=\frac{\beta_{j}}{\beta_{k}}, \mathcal{Z}\left(T, \alpha, \widehat{\beta}_{j}\right)=\int_{u_{j}}^{\infty} \frac{T^{2 / \alpha}}{1+u^{\alpha / 2}} \mathrm{~d} u$, and $u_{j}=$ $\left(\widehat{\beta}_{j} / T\right)^{2 / \alpha}$. Under interference-limited conditions, it simplifies to [14]

$$
\overline{\mathcal{P}}_{c_{P}}=\sum_{k=1}^{K} \frac{\lambda_{k} P_{k}^{2 / \alpha}}{\sum_{j=1}^{K} \lambda_{j} P_{j}^{2 / \alpha} \mathcal{C}_{j}} .
$$

If the HetNet is unbiased i.e. $\widehat{\beta}_{j}=1$ for all $j \in K$, then $\mathcal{C}_{j}=1+\mathcal{Z}(T, \alpha, 1)$ is a constant and $\overline{\mathcal{P}}_{c_{P}}$ becomes

$$
\overline{\mathcal{P}}_{c_{P}}=[1+\mathcal{Z}(T, \alpha, 1)]^{-1}
$$


The average user rate in a HetNet using maximum ABRP connectivity is expressed as [14]

$$
\mathcal{R}_{u_{P}}=\sum_{k=1}^{K} \Xi\left[\pi \lambda_{k} e^{-\frac{\sigma^{2}}{P_{k} L}\left(2^{t}-1\right) z^{\alpha / 2}} e^{-\pi \sum_{j=1}^{K} \lambda_{j} \widehat{P}_{j}^{2 / \alpha} \mathcal{D}_{j}(t) z}\right],
$$

where $\mathcal{D}_{j}(t)=\widehat{\beta}_{j}^{2 / \alpha}+\mathcal{Z}\left(t, \alpha, \widehat{\beta}_{j}\right)$ and $\mathcal{Z}(\cdot)=\left(2^{t}-1\right)^{2 / \alpha} \int_{u_{j}}^{\infty} \frac{1}{1+u^{\alpha / 2}} \mathrm{~d} t$ where $u_{j}=\left(\frac{\widehat{\beta}_{j}}{2^{t}-1}\right)^{2 / \alpha}$. Under interference-limited conditions, it becomes [14]

$$
\overline{\mathcal{R}}_{u_{P}}=\sum_{k=1}^{K} \int_{t>0} \frac{\lambda_{k} P_{k}^{2 / \alpha}}{\sum_{j=1}^{K} \lambda_{j} P_{j}^{2 / \alpha} \mathcal{D}_{j}(t)} \mathrm{d} t .
$$

If the HetNet is unbiased, $\overline{\mathcal{R}}_{u_{P}}$ further simplifies to

$$
\overline{\mathcal{R}}_{u_{P}}=\int_{t>0} \frac{1}{\mathcal{D}(t)} \mathrm{d} t
$$

where $\mathcal{D}(t)=1+\mathcal{Z}(t, \alpha, 1)$ is a constant.

According to (10) and (13), when the HetNet is unbiased, both $\overline{\mathcal{P}}_{c_{P}}$ and $\overline{\mathcal{R}}_{u_{P}}$ are independent of the tuple $\left(K,\left\{\lambda_{j}\right\},\left\{P_{j}\right\}\right)$ which means that operators can densify the HetNet with any number and kinds of BSs without affecting coverage probability and average user rate. The intuitive explanation is that although densification improves average received signal strength, aggregate interference also increases in equal measure [14], [23]. If the HetNet is biased however, all these parameters will influence both $\overline{\mathcal{P}}_{c_{P}}$ and $\overline{\mathcal{R}}_{u_{P}}$.

For maximum i-SINR scheme, the coverage probability can be expressed as [24], [25]

$$
\mathcal{P}_{c_{S}}=\sum_{k=1}^{K} \pi \lambda_{k} \int_{z>0} e^{-\frac{T \sigma^{2}}{P_{k} L} z^{\alpha / 2}} e^{-T^{2 / \alpha} \varrho(\alpha) \sum_{j=1}^{K} \lambda_{j} \widehat{P}_{j}^{2 / \alpha} z} \mathrm{~d} z,
$$

where $\varrho(\alpha)=2 \pi^{2} \alpha^{-1} \csc (2 \pi / \alpha)$. When the HetNet is interference-limited, it simplifies to [24]

$$
\overline{\mathcal{P}}_{c_{S}}=\frac{\pi T^{-2 / \alpha}}{\varrho(\alpha)} \stackrel{(a)}{=} \frac{2}{\pi \sqrt{T}}
$$

where $(a)$ refers to the special case of $\alpha=4$.

Furthermore, the average user rate with maximum i-SINR scheme is expressed as [24], [25]

$$
\mathcal{R}_{u_{S}}=\sum_{k=1}^{K} \Xi\left[\pi \lambda_{k} e^{-\frac{\sigma^{2}}{P_{k} L}\left(2^{t}-1\right) z^{\alpha / 2}} \times e^{-\left(2^{t}-1\right)^{2 / \alpha} \varrho(\alpha) \sum_{j=1}^{K} \lambda_{j} \widehat{P}_{j}^{2 / \alpha} z}\right] .
$$

When the HetNet is interference-limited, it simplifies to [24]

$$
\overline{\mathcal{R}}_{u_{S}}=\frac{\pi}{\varrho(\alpha)} \int_{t>0}\left(2^{t}-1\right)^{-2 / \alpha} \mathrm{d} t .
$$

Therefore, both $\overline{\mathcal{P}}_{c_{S}}$ and $\overline{\mathcal{R}}_{u_{S}}$ are also independent of the tuple $\left(K,\left\{\lambda_{j}\right\},\left\{P_{j}\right\}\right)$, similar to maximum ABRP connectivity. This independence is because users associate with their most favorable BSs in terms of instantaneous SINR. 
We now analyze and express the performance of a HetNet using minimum BTD connectivity in a form that is directly comparable to both maximum ABRP and maximum i-SINR connectivity schemes. The analysis of this scheme in [15] uses another approach and no numerical results are presented to describe its performance. Moreover, we also analyze its optimal deployment configuration in this paper.

Lemma 1. In a K-tier HetNet using minimum BTD connectivity, the $k$-th tier association probability is expressed as

$$
\mathcal{A}_{k}=\frac{\lambda_{k}}{\sum_{j=1}^{K} \lambda_{j}\left(\frac{v_{k}}{v_{j}}\right)^{2}} \text {. }
$$

Proof. See Appendix VII-A.

According to Lemma 1, more users connect to a tier with a higher BS density and a smaller bias value. This is intuitive because a smaller bias value makes BSs of that tier appear to be closer than those of a tier with a larger bias value.

Lemma 2. The distance between a typical user and its serving BS is a random variable whose PDF is expressed as

$$
f_{X_{k}}(x)=\frac{2 \pi \lambda_{k}}{\mathcal{A}_{k}} x e^{-\pi \sum_{j=1}^{K} \lambda_{j}\left(\frac{v_{k}}{v_{j}}\right)^{2} x^{2}}
$$

Proof. See Appendix VII-B.

Theorem 1. The coverage probability of a typical user in a HetNet with minimum BTD connectivity is expressed as

$$
\mathcal{P}_{c_{D}}=\sum_{k=1}^{K} \pi \lambda_{k} \int_{z>0} e^{-\frac{T \sigma^{2}}{P_{k} L} z^{\alpha / 2}} e^{-\pi \sum_{j=1}^{K} \lambda_{j} \widehat{P}_{j}^{2 / \alpha} \mathcal{E}_{j} z} \mathrm{~d} z
$$

where $\mathcal{E}_{j}=\mathcal{S}_{j}+\frac{\widehat{v}_{j}^{-2}}{\widehat{P}_{j}^{2 / \alpha}}, \widehat{v}_{j}=\frac{v_{j}}{v_{k}}, \mathcal{S}_{j}=T^{2 / \alpha} \int_{u_{j}}^{\infty} \frac{1}{1+u^{\alpha / 2}} \mathrm{~d} u$ and $u_{j}=\frac{\widehat{\widehat{v}}_{j}^{-2}}{\left(\widehat{P}_{j} T\right)^{2 / \alpha}}$.

Proof. See Appendix VII-C.

Corollary 1. If the HetNet is interference-limited, coverage probability simplifies to

$$
\overline{\mathcal{P}}_{c_{D}}=\sum_{k=1}^{K} \frac{\lambda_{k} P_{k}^{2 / \alpha}}{\sum_{j=1}^{K} \lambda_{j} P_{j}^{2 / \alpha} \mathcal{E}_{j}} .
$$


When $\left\{v_{j}\right\}=1, \mathcal{E}_{j}=\widehat{P}_{j}^{-2 / \alpha}+\int_{\left(\widehat{P}_{j} T\right)^{-2 / \alpha}}^{\infty} \frac{T^{2 / \alpha}}{1+u^{\alpha / 2}} \mathrm{~d} u$.

Proof. Let $\sigma^{2}=0$ in (20) and solve the resulting integral.

In the unbiased interference-limited HetNet, $\mathcal{E}_{j}$ is not a constant since it also depends on $\widehat{P}_{j}$ (in a typical HetNet, $\widehat{P}_{j} \neq 1$ since BSs in different tiers transmit different power). Therefore, coverage probability depends on the tuple $\left(K,\left\{\lambda_{j}\right\},\left\{P_{j}\right\}\right)$ which contrasts with maximum ABRP scheme in (10). This dependence is a consequence of forcing users to associate with their closest BSs rather than BSs that provide the best signal strength or instantaneous SINR.

Theorem 2. Average user rate in a HetNet using minimum BTD connectivity is expressed as

$$
\mathcal{R}_{u_{D}}=\sum_{k=1}^{K} \Xi\left[\pi \lambda_{k} e^{-\frac{\sigma^{2}}{P_{k} L}\left(2^{t}-1\right) z^{\alpha / 2}} e^{-\pi \sum_{j=1}^{K} \lambda_{j} \widehat{P}_{j}^{2 / \alpha} \mathcal{G}_{j} z}\right],
$$

where $\mathcal{G}_{j}(t)=\mathcal{U}_{j}(t)+\frac{\widehat{v}_{j}^{-2}}{\widehat{P}_{j}^{2 / \alpha}}, \mathcal{U}_{j}(t)=\int_{u_{j}(t)}^{\infty} \frac{\left(2^{t}-1\right)^{2 / \alpha}}{1+u^{\alpha / 2}} \mathrm{~d} u$ and $u_{j}(t)=\frac{\widehat{v}_{j}^{-2}}{\left(\widehat{P}_{j}\left(2^{t}-1\right)\right)^{2 / \alpha}}$.

Proof. See Appendix VII-D.

Corollary 2. If the HetNet is interference-limited, average user rate simplifies to

$$
\overline{\mathcal{R}}_{u_{D}}=\sum_{k=1}^{K} \int_{t>0} \frac{\lambda_{k} P_{k}^{2 / \alpha}}{\sum_{j=1}^{K} \lambda_{j} P_{j}^{2 / \alpha} \mathcal{G}_{j}(t)} \mathrm{d} t .
$$

Proof. Let $\sigma^{2}=0$ in (22) and solve the resulting integral.

Similarly when $\left\{\widehat{v}_{j}\right\}=1, \mathcal{G}_{j}(t)$ is still not a constant since it also depends on the set $\left\{\widehat{P}_{j}\right\}$.Therefore, $\overline{\mathcal{R}}_{u_{D}}$ always depends on the tuple $\left(K,\left\{\lambda_{j}\right\},\left\{P_{j}\right\}\right)$ which contrasts with maximum ABRP connectivity (see $\overline{\mathcal{R}}_{u_{P}}$ in (13)).

Lemma 3. In a mobile environment where $\alpha>2$ and $\sigma^{2}>0$, both coverage probability and average user rate increase monotonically with the $B S$ density $\lambda_{k}$ and transmit power $P_{k}$.

Proof. We consider the expressions for maximum ABRP connectivity to prove Lemma 3 but the proof can easily be extended to the other association schemes. First, consider the variation of coverage probability with the BS density. Assume that a typical user is associated to the $k$-th tier and consider two $k$-th tier BS densities $\lambda_{k_{1}}$ and $\lambda_{k_{2}}$, where $\lambda_{k_{2}}>\lambda_{k_{1}}$. According to (8), the coverage probability corresponding to the BS density $\lambda_{k_{2}}$ is expressed as

$$
\mathcal{P}_{c_{P}}\left(\lambda_{k_{2}}\right)=\sum_{k=1}^{K} \pi \lambda_{k_{2}} \int_{0}^{\infty} e^{-a_{1} z^{\alpha / 2}} e^{-\pi\left(\lambda_{k_{2}} \mathcal{C}+a_{2}\right) z} \mathrm{~d} z,
$$


where $a_{1}=\frac{T \sigma^{2}}{P_{k} L}, a_{2}=\sum_{j=1, j \neq k}^{K} \lambda_{j} \widehat{P}_{j}^{2 / \alpha} \mathcal{C}_{j}$, where $\mathcal{C}_{j}=\mathcal{C}=1+\mathcal{Z}(T, \alpha, 1)$ is a constant. Substituting $z=x \frac{\lambda_{k_{1}}}{\lambda_{k_{2}}}$ into (24),

$$
\begin{aligned}
\mathcal{P}_{c_{P}}\left(\lambda_{k_{2}}\right) & =\sum_{k=1}^{K} \pi \lambda_{k_{1}} \int_{0}^{\infty} e^{-a_{1}\left(\frac{\lambda_{k_{1}}}{\lambda_{k_{2}}}\right)^{\alpha / 2} x^{\alpha / 2}} \times e^{-\pi\left(\lambda_{k_{1}} \mathcal{C}+a_{2} \frac{\lambda_{k_{1}}}{\lambda_{k_{2}}}\right) x} \mathrm{~d} x \\
& \stackrel{(b)}{>} \sum_{k=1}^{K} \pi \lambda_{k_{1}} \int_{0}^{\infty} e^{-a_{1} x^{\alpha / 2}} e^{-\pi\left(\lambda_{k_{1}} \mathcal{C}+a_{2}\right) x} \mathrm{~d} x \\
& =\mathcal{P}_{c_{P}}\left(\lambda_{k_{1}}\right)
\end{aligned}
$$

where $(b)$ follows since $a_{1}>0, a_{2}>0, \mathcal{C}>0, \alpha>2$, and $\left(\lambda_{k_{1}} / \lambda_{k_{2}}\right)<1$. Therefore, the coverage probability always increases if the BS density is increased from $\lambda_{k_{1}}$ to $\lambda_{k_{2}}$. Since $a_{2}>0$ in biased HetNets, the proof still holds. The dependence of average user rate in (11) on the BS density can easily be proved following the same procedure.

To show dependence on transmit power, we use the average user rate expression in (11). Consider two transmit power levels $P_{k_{1}}$ and $P_{k_{2}}$ where $P_{k_{2}}>P_{k_{1}}$. For $P_{k_{2}}$, (11) becomes

$$
\mathcal{R}_{u_{P}}\left(P_{k_{2}}\right)=\sum_{k=1}^{K} \Xi\left[\pi \lambda_{k} e^{-\left(b_{1} / P_{k_{2}}\right) z^{\alpha / 2}} e^{-\left(b_{2} / P_{k_{2}}^{2 / \alpha}\right) z}\right],
$$

where $b_{1}=\frac{\sigma^{2}}{L}\left(2^{t}-1\right)$ and $b_{2}=\pi \sum_{j=1}^{K} \lambda_{j} P_{j}^{2 / \alpha} \mathcal{D}_{j}(t)$. Similarly for $P_{k_{1}}$, (11) is rewritten as

$$
\mathcal{R}_{u_{P}}\left(P_{k_{1}}\right)=\sum_{k=1}^{K} \Xi\left[\pi \lambda_{k} e^{-\left(b_{1} / P_{k_{1}}\right) z^{\alpha / 2}} e^{-\left(b_{2} / P_{k_{1}}^{2 / \alpha}\right) z}\right] .
$$

Since $b_{1}>0, b_{2}>0, \alpha>2$ and $P_{k_{2}}>P_{k_{1}}$, both exponential terms in (25) are larger than their corresponding terms in (26) which confirms that $\mathcal{R}_{u_{P}}\left(P_{k_{2}}\right)>\mathcal{R}_{u_{P}}\left(P_{k_{1}}\right)$. The dependence of coverage (8) on transmit power can easily be proved following the same procedure.

\section{HetNet Deployment Optimization}

The previous section presented the performance analysis of a general $K$-tier HetNet in terms of its coverage probability and average user rate for different cell association schemes. In this section, these results will be used in the optimization of the HetNet to devise its optimal deployment strategy in terms of the deployment factor [20]. The deployment factor, expressed

as $\mathcal{H}=\sum_{j=1}^{K} \lambda_{j} P_{j}^{2 / \alpha}$, essentially combines in some mathematical form the BS densities and transmit powers per tier that jointly achieve a given performance objective. In this paper, the objective is to minimize the APC of the HetNet subject to coverage and rate constraints. Therefore, the deployment factor can be optimized to determine the specific optimal BS densities and transmit powers per tier that minimize the APC. 


\section{A. Coverage Probability Constraint}

Theorem 3. The coverage probability of a typical user in a HetNet with maximum ABRP connectivity is approximated as

$$
\mathcal{P}_{c_{P}} \approx \overline{\mathcal{P}}_{c_{P}}-T \sigma^{2} \psi(\alpha) \sum_{k=1}^{K} \frac{\lambda_{k} P_{k}^{2 / \alpha}}{\left[\sum_{j=1}^{K} \lambda_{j} P_{j}^{2 / \alpha} \mathcal{C}_{j}\right]^{\frac{\alpha}{2}+1}}
$$

where $\psi(\alpha)=\frac{\Gamma\left(\frac{\alpha}{2}+1\right)}{\pi^{\alpha / 2} L}$ and $\Gamma(\cdot)$ is the gamma function.

Proof. Over a realistic SNR range, $T \sigma^{2} \ll P_{k} L$. Hence the first exponential term in (8) can be approximated as

$$
e^{-\frac{T \sigma^{2}}{P_{k} L} z^{\alpha / 2}} \approx 1-\frac{T \sigma^{2}}{P_{k} L} z^{\alpha / 2}
$$

Therefore, substituting (28) into (8) gives

$$
\mathcal{P}_{c_{P}} \approx \sum_{k=1}^{K} \pi \lambda_{k}\left[\int_{z>0} e^{-\pi \sum_{j=1}^{K} \lambda_{j} \widehat{P}_{j}^{2 / \alpha} \mathcal{C}_{j} z} \mathrm{~d} z-\frac{T \sigma^{2}}{P_{k} L} \int_{z>0} z^{\alpha / 2} e^{-\pi \sum_{j=1}^{K} \lambda_{j} \widehat{P}_{j}^{2 / \alpha} \mathcal{C}_{j} z} \mathrm{~d} z\right] .
$$

Solving both integrals gives the result.

The approximated coverage probability in (27) will be used to define one of the constraints in the optimization of the HetNet to devise its optimal deployment factor. To ensure high accuracy of (28), the value of $T$ should be reasonably small, which is normally the case for a practical SINR coverage constraint. The accuracy of the approximated coverage probability and average user rate is verified in Fig. 1. The accuracy of this approximation on other schemes is equally high; for instance see Fig. 4 for the minimum BTD scheme. Moreover, all these illustrations prove that the accuracy improves with the BS density which is important since future networks will be very dense [8].

1) Unbiased HetNet: When $\left\{\beta_{j}\right\}=1$, then $\left\{\mathcal{C}_{j}\right\}=\mathcal{C}$, where $\mathcal{C}=1+\mathcal{Z}(T, \alpha, 1)$ is a constant. Therefore, (27) can further be simplified to

$$
\mathcal{P}_{c_{P}} \approx \overline{\mathcal{P}}_{c_{P}}-\frac{T \sigma^{2} \psi(\alpha)}{\mathcal{C}^{\frac{\alpha}{2}+1}\left[\sum_{j=1}^{K} \lambda_{j} P_{j}^{2 / \alpha}\right]^{\alpha / 2}}
$$

Using $\mathcal{P}_{c_{P}}=\epsilon \overline{\mathcal{P}}_{c_{P}}$ and Lemma 3 , the coverage probability constraint can be rewritten in terms of the optimal deployment factor $\mathcal{H}_{c}^{\star}$ as follows

$$
\mathcal{H}_{c}^{\star} \equiv \sum_{j=1}^{K} \lambda_{j} P_{j}^{2 / \alpha}=\frac{1}{\mathcal{C}}\left(\frac{T \sigma^{2} \psi(\alpha)}{1-\epsilon}\right)^{2 / \alpha}
$$


Therefore, for an unbiased HetNet using maximum ABRP connectivity, $\mathcal{H}_{c}^{\star}$ is expressed in closed form and it is an increasing function of $T, \sigma^{2}$ and $\epsilon$.

To illustrate, consider a typical $K$-tier unbiased HetNet defined by the parameters $K=2$, $\lambda_{j} \in\left\{\lambda_{b}, \lambda_{s}\right\}$ and $P_{j} \in\left\{P_{b}, P_{s}\right\}$. The optimal deployment factor is expressed as $\mathcal{H}_{c}^{\star}=\lambda_{b} P_{b}^{2 / \alpha}+$ $\lambda_{s} P_{s}^{2 / \alpha}$. This special case of a two-tier HetNet using maximum ABRP connectivity is investigated in [20] although $\mathcal{H}_{c}^{\star}$ is not expressed in closed form. A network APC minimization framework is formulated to determine the optimal macro BS and micro BS densities and their respective transmit powers subject to a coverage constraint only.

2) Biased Network: In this case, $\left\{\mathcal{C}_{j}\right\} \neq \mathcal{C}$ and further simplification of (27) is not possible. Instead, using $\mathcal{P}_{c_{P}}=\epsilon \overline{\mathcal{P}}_{c_{P}}$, (27) can be rewritten as

$$
(1-\epsilon) \overline{\mathcal{P}}_{c_{P}}-T \sigma^{2} \psi(\alpha) \sum_{k=1}^{K} \frac{\lambda_{k} P_{k}^{2 / \alpha}}{\mathcal{Q}_{c}^{\frac{\alpha}{2}+1}}=0,
$$

where $\mathcal{Q}_{c}=\sum_{j=1}^{K} \lambda_{j} P_{j}^{2 / \alpha} \mathcal{C}_{j}$. Due to the variation of $\mathcal{C}_{j}$ with the $\{k, j\}$ pair, it is not possible to isolate and express the deployment factor $\mathcal{H}_{c}=\sum_{j=1}^{K} \lambda_{j} P_{j}^{2 / \alpha}$ in closed form.

In order to investigate the impact of biasing on the deployment factor, consider a conventional two-tier biased HetNet and assume that the deployment factor of the macrocell tier, denoted as $\mathcal{H}_{b}=\lambda_{b} P_{b}^{2 / \alpha}$, is known. Note that this is often the case for an operator that has an existing network of macro BSs and seeks to densify it with small BSs for targeted coverage and capacity enhancements. Hence $\mathcal{Q}_{c}=\mathcal{H}_{b} \mathcal{C}_{b}+\mathcal{H}_{s, c}^{\star} \mathcal{C}_{s}$ where $\mathcal{H}_{s, c}^{\star}=\lambda_{s} P_{s}^{2 / \alpha}$ is the required optimal small cell tier deployment factor that jointly satisfies the coverage constraint. Since $\mathcal{H}_{b}$ is known, the value of $\mathcal{H}_{s, c}^{\star}$ that satisfies (32) can be determined easily using numerical methods such as the bisection method [30]. However, the required value of $\mathcal{H}_{s, c}^{\star}$ is affected by the bias ratio $\beta$, defined as $\beta=\beta_{s} / \beta_{b}$. The bias ratio can therefore be varied to investigate the effect of biasing on the energy performance of the two-tier HetNet.

To determine the specific optimal values of the BS density and transmit power of the small cell tier that minimize the HetNet APC, $\mathcal{H}_{s, c}^{\star}$ is optimized as a bivariate optimization problem according to the following framework:

$$
\begin{cases}\underset{\lambda_{s, c}, P_{s}}{\operatorname{minimize}} & \mathrm{APC}=\lambda_{s, c}\left(N_{s} P_{0, s}+\Delta_{s} P_{s}\right) \\ \text { subject to } & \lambda_{s, c} P_{s}^{2 / \alpha}=\mathcal{H}_{s, c}^{\star} \\ & 0 \leq P_{s} \leq \bar{P}_{s},\end{cases}
$$


where $\bar{P}_{s}$ is the maximum small BS transmit power. This problem can easily be converted to a single-variable problem, and its solutions are determined as

$$
P_{s}^{\star}=\min \left\{\frac{2 N_{s} P_{0, s}}{\Delta_{s}(\alpha-2)}, \bar{P}_{s}\right\}, \lambda_{s, c}^{\star}=\mathcal{H}_{s, c} P_{s}^{\star-2 / \alpha} .
$$

\section{B. Average User Rate Constraint}

Theorem 4. The average user rate in a HetNet with maximum ABRP connectivity can be approximated as

$$
\mathcal{R}_{u_{P}} \approx \overline{\mathcal{R}}_{u_{P}}-\sigma^{2} \psi(\alpha) \sum_{k=1}^{K} \int_{t>0} \frac{\lambda_{k} P_{k}^{2 / \alpha}\left(2^{t}-1\right)}{\left[\sum_{j=1}^{K} \lambda_{j} P_{j}^{2 / \alpha} \mathcal{D}_{j}(t)\right]^{\frac{\alpha}{2}+1}} \mathrm{~d} t .
$$

Proof. Over a realistic SNR range, the first exponential term in (11) can be approximated as

$$
e^{-\frac{\sigma^{2}}{P_{k} L}\left(2^{t}-1\right) z^{\alpha / 2}} \approx 1-\frac{\sigma^{2}}{P_{k} L}\left(2^{t}-1\right) z^{\alpha / 2}
$$

Substituting (36) into (11) gives

$$
\mathcal{R}_{u_{P}} \approx \sum_{k=1}^{K} \pi \lambda_{k}\left\{\Xi\left[e^{-\pi \sum_{j=1}^{K} \lambda_{j} \tilde{P}_{j}^{2 / \alpha} \mathcal{D}_{j}(t) z}\right]-\Xi\left[\frac{\sigma^{2}}{P_{k} L}\left(2^{t}-1\right) z^{\alpha / 2} e^{-\pi \sum_{j=1}^{K} \lambda_{j} \tilde{P}_{j}^{2 / \alpha} \mathcal{D}_{j}(t) z}\right]\right\} .
$$

Solving both integrals gives the result.

Similar to the coverage probability case, the approximated average user rate is highly accurate and its accuracy increases with the BS density as verified in Fig. 2. Note that the other schemes are also highly accurate; for instance see Fig. 3 for the maximum i-SINR association scheme.

1) Unbiased Network: In this case, $\mathcal{D}_{j}(t)=\mathcal{D}(t), \forall j \in K$, where $\mathcal{D}(t)=1+\mathcal{Z}(t, \alpha, 1)$ is a constant. Therefore, (35) can be further simplified to

$$
\mathcal{R}_{u_{P}}=\overline{\mathcal{R}}_{u_{P}}-\frac{\sigma^{2} \psi(\alpha)}{\left[\sum_{j=1}^{K} \lambda_{j} P_{j}^{2 / \alpha}\right]^{\alpha / 2}} \int_{t>0} \frac{2^{t}-1}{\mathcal{D}(t)^{\frac{\alpha}{2}+1}} \mathrm{~d} t .
$$

Using $\mathcal{R}_{u_{P}}=\kappa \overline{\mathcal{R}}_{u_{P}}$ and Lemma 3, (37) can be rewritten as

$$
\mathcal{H}_{r}^{\star} \equiv \sum_{j=1}^{K} \lambda_{j} P_{j}^{2 / \alpha}=\left(\frac{\sigma^{2} \psi(\alpha) g(t, \alpha)}{1-\kappa}\right)^{2 / \alpha}
$$

where $g(t, \alpha)=\int_{t>0} \frac{2^{t}-1}{\mathcal{D}(t)^{\frac{\alpha}{2}+1}} \mathrm{~d} t \times\left(\int_{t>0} \frac{1}{\mathcal{D}(t)} \mathrm{d} t\right)^{-1}$ and $\mathcal{H}_{r}^{\star}$ is the optimal deployment factor. Hence $\mathcal{H}_{r}^{\star}$ is also expressed in closed form and it is an increasing function of $\kappa$ and $\sigma^{2}$. 
2) Biased Network: In this case, it is not possible to isolate the term $\sum_{j=1}^{K} \lambda_{j} P_{j}^{2 / \alpha}$ in (35). Therefore, the average user rate constraint can be rewritten as

$$
(1-\kappa) \overline{\mathcal{R}}_{u_{P}}-\sigma^{2} \psi \sum_{k=1}^{K} \int_{t>0} \frac{\lambda_{k} P_{k}^{2 / \alpha}\left(2^{t}-1\right)}{\mathcal{Q}_{r}^{\frac{\alpha}{2}+1}} \mathrm{~d} t=0,
$$

where $\mathcal{Q}_{r}=\sum_{j=1}^{K} \lambda_{j} P_{j}^{2 / \alpha} \mathcal{D}_{j}(t)$. Consider a conventional two-tier HetNet and assume the same macrocell tier deployment factor $\mathcal{H}_{b}$. Then $\mathcal{Q}_{r}=\mathcal{H}_{b} \mathcal{D}_{b}(t)+\mathcal{H}_{s, r}^{\star} \mathcal{D}_{s}(t)$ where $\mathcal{H}_{s, r}^{\star}=\lambda_{s} P_{s}^{2 / \alpha}$ is the optimal small cell tier deployment factor that jointly satisfies the rate constraint. In this case, $\mathcal{H}_{s, r}^{\star}$ is also influenced by the biasing in the HetNet and this is investigated by varying the bias ratio $\beta$. The optimization of $\mathcal{H}_{s, r}^{\star}$ to minimize APC is similar to the procedure in (33)-(34). The solutions are expressed as

$$
P_{s}^{\star}=\min \left\{\frac{2 N_{s} P_{0, s}}{\Delta_{s}(\alpha-2)}, \bar{P}_{s}\right\}, \quad \lambda_{s, r}^{\star}=\mathcal{H}_{s, r}^{\star} P_{s}^{\star-2 / \alpha} .
$$

Therefore, according to (34) and (40), the optimal transmit power $P_{s}^{\star}$ subject to both coverage probability and average user rate constraints is similar. Furthermore, if the type of deployed small BSs is known, the optimal transmit power can be predetermined.

\section{Maximum ABRP Connectivity: Overall Solution}

Since the coverage probability and average rate constraints are complementary to each other (i.e. optimization based on one measure also improves the other measure), the optimal solution is one that satisfies both constraints. Therefore, we separate the optimization problem based on both constraints into two separate optimization problems, one constrained by coverage probability and the other by the average user rate. The overall optimal solution will be the maximum of the two individual solutions i.e. the optimal small BS density is $\lambda_{s}^{\star}=\max \left\{\lambda_{s, c}^{\star}, \lambda_{s, r}^{\star}\right\}$ and $P_{s}^{\star}$ is the optimal transmit power. The resulting APC at optimal configuration is evaluated as

$$
\mathrm{APC}=\lambda_{b}\left(N_{b} P_{0, b}+\Delta_{b} P_{b}\right)+\lambda_{s}^{\star}\left(N_{s} P_{0, s}+\Delta_{s} P_{s}^{\star}\right) .
$$

Furthermore, according to (32) and (39), both $\mathcal{H}_{s, c}$ and $\mathcal{H}_{s, r}$ are influenced by the value of $\beta$ in the two-tier HetNet. Denote the overall optimal small cell tier deployment factor as $\mathcal{H}_{s}^{\star}=\max \left\{\mathcal{H}_{s, c}^{\star}, \mathcal{H}_{s, r}^{\star}\right\}$. Then the optimal $\beta$ at which $\mathcal{H}_{s}^{\star}$ is minimized is determined using

$$
\begin{cases}\underset{\beta}{\operatorname{minimize}} & \max \left\{\mathcal{H}_{s, c}^{\star}, \mathcal{H}_{s, r}^{\star}\right\} \\ \text { subject to } & \lambda_{s, c}^{\star} \geq 0, \lambda_{s, r}^{\star} \geq 0, \\ & \beta>0, P_{s}^{\star}>0 .\end{cases}
$$


Operating the HetNet at its optimal $\beta^{\star}$ minimizes the APC.

\section{HetNets with Minimum BTD Connectivity}

Theorem 5. The coverage probability of a typical user in a HetNet with minimum BTD connectivity is approximated as

$$
\mathcal{P}_{c_{D}} \approx \overline{\mathcal{P}}_{c_{D}}-T \sigma^{2} \psi(\alpha) \sum_{k=1}^{K} \frac{\lambda_{k} P_{k}^{2 / \alpha}}{\left[\sum_{j=1}^{K} \lambda_{j} P_{j}^{2 / \alpha} \mathcal{E}_{j}\right]^{\frac{\alpha}{2}+1}}
$$

Proof. Substitute (28) into (20) and solve the integrals.

Since the term $\mathcal{E}_{j}$ depends on $\widehat{P}_{j}$, further simplification is not possible even in the case of the unbiased HetNet. Using $\mathcal{P}_{c_{D}} \geq \epsilon \overline{\mathcal{P}}_{c_{D}}$, (43) is instead rewritten as

$$
(1-\epsilon) \overline{\mathcal{P}}_{c_{D}}-T \sigma^{2} \psi(\alpha) \sum_{k=1}^{K} \frac{\lambda_{k} P_{k}^{2 / \alpha}}{\mathcal{Q}_{c}^{\frac{\alpha}{2}+1}} \geq 0,
$$

where $\mathcal{Q}_{c}=\sum_{j=1}^{K} \lambda_{j} P_{j}^{2 / \alpha} \mathcal{E}_{j}$. Similar to maximum ABRP connectivity, consider the same twotier HetNet whose macrocell tier deployment factor $\mathcal{H}_{b}$ is known. Then, $\mathcal{Q}_{c}=\mathcal{H}_{b} \mathcal{E}_{b}+\mathcal{H}_{s, c}^{\star} \mathcal{E}_{s}$. The value of $\mathcal{H}_{s, c}^{\star}$ is determined using numerical methods and its optimization to determine the optimal $\lambda_{s, c}^{\star}$ and $P_{s}^{\star}$ follows the same procedure shown in (33)-(34).

Theorem 6. The average rate of a typical user in a HetNet with minimum BTD connectivity is approximated as

$$
\mathcal{R}_{u_{D}} \approx \overline{\mathcal{R}}_{u_{D}}-\sigma^{2} \psi(\alpha) \sum_{k=1}^{K} \int_{t>0} \frac{\lambda_{k} P_{k}^{2 / \alpha}\left(2^{t}-1\right)}{\left[\sum_{j=1}^{K} \lambda_{j} P_{j}^{2 / \alpha} \mathcal{G}_{j}(t)\right]^{\frac{\alpha}{2}+1}} \mathrm{~d} t .
$$

Proof. Substitute (36) into (22) and solve the integrals.

Using $\mathcal{R}_{u_{D}} \geq \kappa \overline{\mathcal{R}}_{u_{D}}$, the average rate can be rewritten as

$$
(1-\kappa) \overline{\mathcal{R}}_{u_{D}}-\sigma^{2} \psi(\alpha) \sum_{k=1}^{K} \frac{\lambda_{k} P_{k}^{2 / \alpha}\left(2^{t}-1\right)}{\mathcal{Q}_{r}^{\frac{\alpha}{2}+1}} \geq 0,
$$

where $\mathcal{Q}_{r}=\sum_{j=1}^{K} \lambda_{j} P_{j}^{2 / \alpha} \mathcal{G}_{j}(t)$. Since $\mathcal{G}_{j}(t)$ also depends on $\widehat{P}_{j}$, it is not constant even in the unbiased HetNet and a closed form expression of the deployment factor is not possible. Assuming a two-tier HetNet in which $\mathcal{H}_{b}$ is known, then $\mathcal{Q}_{r}=\mathcal{H}_{b} \mathcal{G}_{b}(t)+\mathcal{H}_{s, r}^{\star} \mathcal{G}_{s}(t)$. The value of $\mathcal{H}_{s, r}^{\star}$ can be determined by numerical methods and its optimization follows (33)-(34). The optimal BS density $\lambda_{s}^{\star}$ is obtained as $\lambda_{s}^{\star}=\max \left\{\lambda_{s, c}, \lambda_{s, r}\right\}$ and the transmit power is $P_{s}^{\star}$. 


\section{E. HetNets with Maximum i-SINR Connectivity}

Theorem 7. The coverage probability of a typical user in a HetNet with maximum i-SINR connectivity is approximated as

$$
\mathcal{P}_{c_{S}} \approx \overline{\mathcal{P}}_{c_{S}}-\frac{\pi \sigma^{2} \xi(\alpha) T^{-2 / \alpha}}{\varrho(\alpha)\left[\sum_{j=1}^{K} \lambda_{j} P_{j}^{2 / \alpha}\right]^{\alpha / 2}}
$$

where $\xi(\alpha)=\frac{\Gamma\left(\frac{\alpha}{2}+1\right)}{L \varrho(\alpha)^{\alpha / 2}}$ is a constant.

Proof. Substitute (28) into (14) and solve the integrals.

Therefore, using $\mathcal{P}_{c_{S}} \geq \epsilon \overline{\mathcal{P}}_{c_{S}}$, (47) is rewritten as

$$
\sum_{j=1}^{K} \lambda_{j} P_{j}^{2 / \alpha} \geq\left(\frac{\xi(\alpha) \sigma^{2}}{1-\epsilon}\right)^{2 / \alpha}
$$

According to (48), the deployment factor $\mathcal{H}_{c}$ is independent of $T$ but increases with both $\epsilon$ and $\sigma^{2}$. Using Lemma 3, the optimal deployment factor of the HetNet is expressed in closed form as $\mathcal{H}_{c}^{\star}=\left(\frac{\xi(\alpha) \sigma^{2}}{1-\epsilon}\right)^{2 / \alpha}$.

Theorem 8. The average rate of a typical user in a HetNet with maximum i-SINR connectivity is approximated as

$$
\mathcal{R}_{u_{S}} \approx \overline{\mathcal{R}}_{u_{S}}-\frac{\pi \sigma^{2} \xi(\alpha)}{\varrho(\alpha)\left[\sum_{j=1}^{K} \lambda_{j} P_{j}^{2 / \alpha}\right]^{\alpha / 2}} \int_{t>0}\left(2^{t}-1\right)^{-2 / \alpha} \mathrm{d} t
$$

Proof. Substitute (36) into (16) and solve the integrals.

Using $\mathcal{R}_{u_{S}} \geq \kappa \overline{\mathcal{R}}_{u_{S}}$, (49) is rewritten as

$$
\sum_{j=1}^{K} \lambda_{j} P_{j}^{2 / \alpha} \geq\left(\frac{\xi(\alpha) \sigma^{2}}{1-\kappa}\right)^{2 / \alpha}
$$

Hence, the optimal deployment factor is expressed in closed form as $\mathcal{H}_{r}^{\star}=\left(\frac{\xi(\alpha) \sigma^{2}}{1-\kappa}\right)^{2 / \alpha}$. According to (48) and (50), the separate (optimal) deployment factors of the HetNet based on coverage probability and average user rate constraints are similar when $\epsilon=\kappa$.

\section{NuMERiCAl Results}

Consider a PPP-based conventional two-tier HetNet with the default parameters shown in Table I, unless otherwise stated. 
TABLE I

SimULATION PARAMETERS

\begin{tabular}{|l|l|}
\hline \hline Parameters & Value \\
\hline Network size & $A=10 \mathrm{~km} \times 10 \mathrm{~km}$ \\
\hline System bandwidth & $\mathcal{B}=20 \mathrm{MHz}$ \\
\hline Pathloss parameters & $L=-55 \mathrm{~dB}, \alpha=4$ \\
\hline Additive noise power & $\sigma^{2}=-110 \mathrm{dBm}$ \\
\hline SINR threshold & $T=0 \mathrm{~dB}$ \\
\hline Macrocell tier parameters & $\mathcal{H}_{b}=4 \times 10^{-5}$ Watts $/ \mathrm{m}^{2}, \beta_{b}=0 \mathrm{~dB}$ \\
\hline Optimization constraints & $\epsilon=0.9, \kappa=0.9$ \\
\hline Macro BS power parameters & $N_{b}=6, P_{0, b}=130, \Delta_{b}=4.7$ \\
\hline Small BS power parameters & $N_{s}=2, P_{0, s}=6.8, \Delta_{s}=4.0$ \\
\hline Transmit power limiting values & $P_{b}=43 \mathrm{dBm}, \bar{P}_{s}=21 \mathrm{dBm}$ \\
\hline
\end{tabular}

Figs. 1-2 illustrate the exact and approximated coverage probability and average user rate performances against small cell density in a HetNet using maximum ABRP connectivity. By varying the small cell density, the inter-site distances and interference level are changed accordingly and therefore, the following analysis can also be viewed as the effective impact of these parameters on the resulting network performance. These results verify the increasing accuracy of the approximated coverage probability in (27) and average user rate in (35) as the HetNet tends to its interference-limited scenario.

For maximum i-SINR and minimum BTD association schemes, Figs. 3 and 4 verify the accuracy of their approximated average user rate shown in (49) and coverage probability shown in (43) respectively. All these results show that the approximations utilized in this work are accurate at high BS density where the effect of noise becomes negligible. Therefore, this approximation approach can be used to determine very accurate deployment factors of dense HetNets.

The effect of additive noise is to reduce the coverage and rate performance of the HetNet compared to its interference-limited scenario. As Figs. 1-3 illustrate, when the small BS density increases, both coverage probability and average user rate approach their respective interference-limited performances (upper bounds) $\overline{\mathcal{P}}_{c}$ and $\overline{\mathcal{R}}_{u}$ because the HetNet gradually becomes interference-limited. Further deployment of extra small BSs beyond this point gives no gain in terms of coverage probability or average user rate although they consume energy.

Figs. 1-2 also illustrate that the effect of biasing is to reduce the coverage probability and average user rate of a HetNet using maximum ABRP scheme. This is a consequence of forcing 


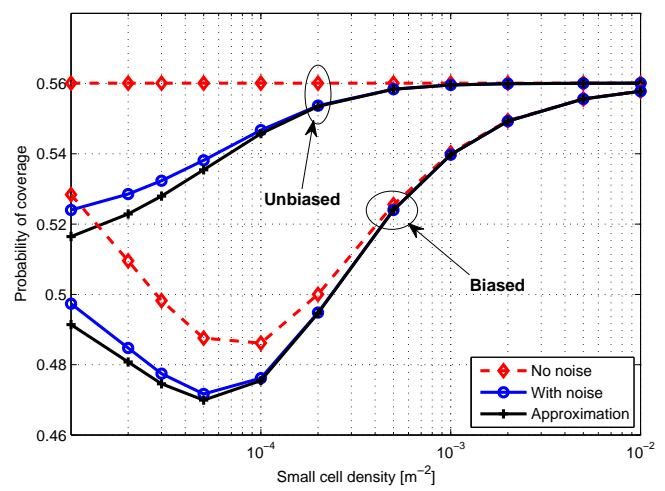

Fig. 1. Coverage probability versus small cell density for a HetNet using maximum ABRP connectivity $(\beta=10 \mathrm{~dB})$.

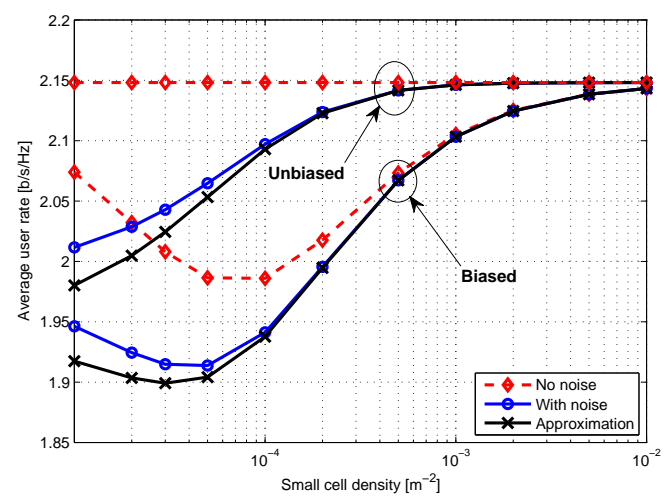

Fig. 2. Average user rate versus small cell density for a HetNet using maximum ABRP connectivity $(\beta=10 \mathrm{~dB})$.

some users to connect to small BSs even though they receive better signal strength from macro BSs. When the small BS density $\left(\lambda_{s}\right)$ is sufficiently low, the macrocell tier overwhelms the small cell tier and the HetNet essentially behaves like a homogeneous network of macro BSs. In this scenario, the effect of biasing on coverage and rate performance gradually fades and the corresponding performance characteristics of the biased HetNet eventually merge with those of the unbiased HetNet. In the low $\lambda_{s}$ regime, increasing $\lambda_{s}$ offloads more users to small BSs but since $\lambda_{s}$ is still low, the received signal strength is also low due to large distances between small BSs and their users but the interference increases. As a result, depending on the bias ratio, the performance may initially drop even as $\lambda_{s}$ increases. Eventually however, the distances between small BSs and their users continue to reduce with increasing $\lambda_{s}$ until such a point that there is a performance gain. In the high $\lambda_{s}$ regime where $\lambda_{s} \gg \lambda_{b}$, the performance of the biased HetNet approaches that of the unbiased HetNet since the small cell tier becomes too dominant 


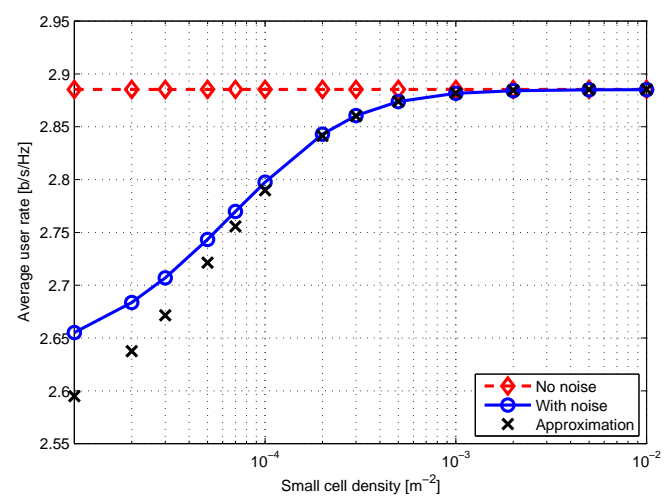

Fig. 3. Verification of the average user rate approximation in a HetNet using maximum i-SINR connectivity $(T=0 \mathrm{~dB})$.

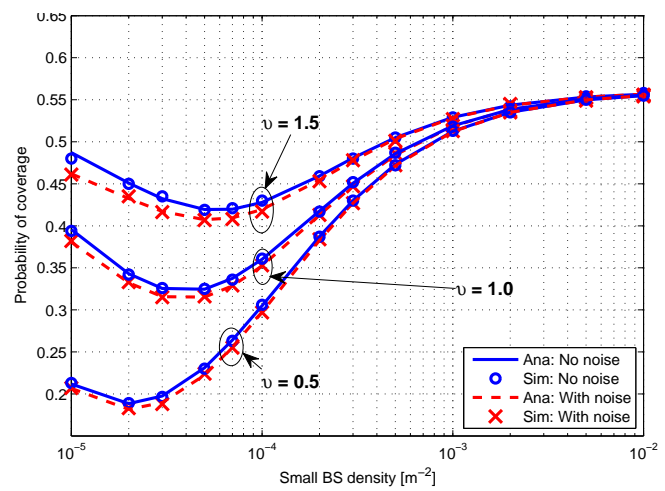

Fig. 4. Verifying coverage probability approximation of the HetNet using minimum BTD connectivity, where $v=v_{s} / v_{b}$.

that it effectively connects most of the users (or covers most of the area). Hence, the HetNet also essentially behaves like a homogenous network of small BSs and biasing has no impact.

For minimum BTD connectivity, when the bias ratio $v>1$ in Fig. 4, it means that the HetNet is actually biased to favour macro BSs instead of small BSs which explains the performance gain over the unbiased HetNet. However, biasing should always favour small BSs over macro BSs to improve load balancing and enhance the average sum rate. Hence, biasing in the practical range of $v<1$ also reduces coverage probability and average rate performance as expected.

Fig. 5 illustrates the variation of average sum rate with small BS density for different values of user density $\lambda_{u}$ in a maximum ABRP-based HetNet. Generally average sum rate increases with $\lambda_{s}$ because as more small BSs are deployed, the average number of users per BS in both tiers reduces which enhances the average bandwidth per user. At low $\lambda_{s}$ (where $\lambda_{u} \gg \lambda_{s}$ ), all BSs in all tiers are highly likely to contain users and the average sum rate increases almost linearly 


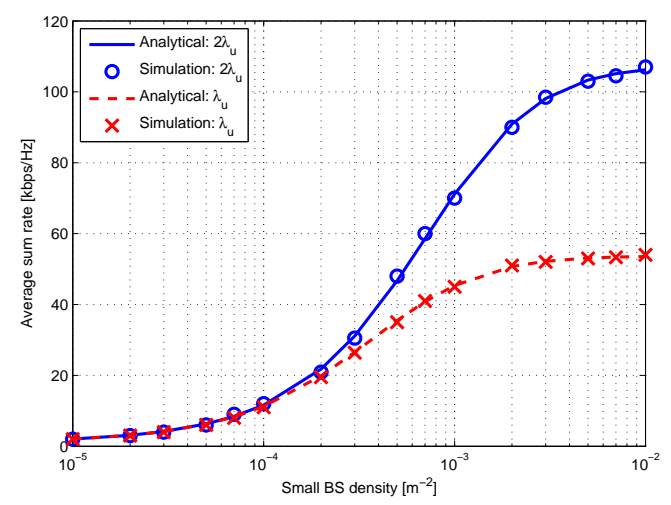

Fig. 5. Average sum rate of the unbiased HetNet using maximum ABRP connectivity $\left(\lambda_{u}=10^{-3} \mathrm{~m}^{-2}\right)$.

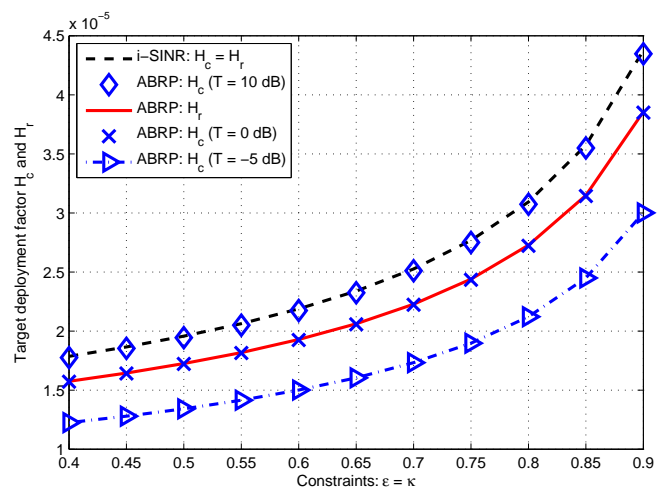

Fig. 6. Deployment factors of the unbiased HetNet using maximum i-SINR and maximum ABRP connectivity schemes.

with the BS density. Increasing the user density does not improve average sum rate since these additional users simply share the same bandwidth. As $\lambda_{s}$ increases further, average bandwidth per user continues to increase which enhances average sum rate. In addition, idle BSs begin to emerge as more small BSs are deployed. Increasing the user density reduces the density of these idle BSs which further enhances the average sum rate. However, the increasing interference begins to limit the average sum rate which eventually saturates in the $\lambda_{s} \gg \lambda_{u}$ regime.

Fig. 6 illustrates the variation of the HetNet deployment factors $\mathcal{H}_{c}$ and $\mathcal{H}_{r}$ with their respective constraint ratios $\epsilon$ and $\kappa$ for both maximum ABRP and maximum i-SINR schemes. Generally both $\mathcal{H}_{c}$ and $\mathcal{H}_{r}$ increase with $\epsilon$ and $\kappa$ respectively because as $\epsilon$ and $\kappa$ increase, the HetNet is required to approach its interference-limited state which requires a higher deployment factor. For maximum i-SINR connectivity, the deployment factors $\mathcal{H}_{c}$ and $\mathcal{H}_{r}$ are equal when $\epsilon=\kappa$ and are both independent of $T$ (see (48) and (50)). For maximum ABRP scheme however, $\mathcal{H}_{c}$ depends 


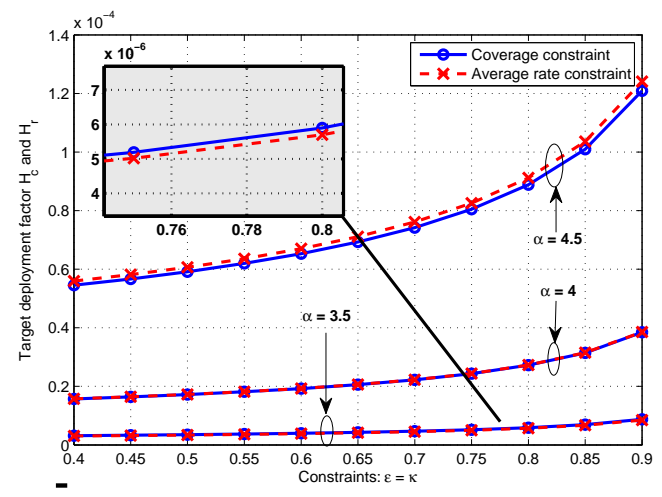

Fig. 7. Deployment factor for the unbiased $K$-tier HetNet using maximum ABRP connectivity $(T=0 \mathrm{~dB})$.

on $T$ but $\mathcal{H}_{r}$ is independent of $T$. Specifically, $\mathcal{H}_{c}$ reduces with $T$ since a lower SINR target can be achieved with a lower deployment factor. When $T=0 \mathrm{~dB}, \mathcal{H}_{c}$ and $\mathcal{H}_{r}$ are approximately equal. However, when $T>0 \mathrm{~dB}, \mathcal{H}_{c}>\mathcal{H}_{r}$ and when $T<0 \mathrm{~dB}, \mathcal{H}_{c}<\mathcal{H}_{r}$. Furthermore, Fig. 7 shows that when $\alpha=4, \mathcal{H}_{r} \approx \mathcal{H}_{c}$; when $\alpha>4, \mathcal{H}_{r}>\mathcal{H}_{c}$; and when $\alpha<4, \mathcal{H}_{c}>\mathcal{H}_{r}$. Note that when $\mathcal{H}_{c}$ dominates $\mathcal{H}_{r}$, it becomes the decisive parameter in the optimization and vice versa. Therefore, the dependence of $\max \left\{\mathcal{H}_{c}, \mathcal{H}_{r}\right\}$ on the combination of values in the set $\{T, \alpha\}$ justifies the necessity of using both coverage probability and average user rate constraints.

In addition, the deployment factors $\mathcal{H}_{c}$ of both maximum ABRP and maximum i-SINR schemes are approximately equal when $T=10 \mathrm{~dB}$ as shown in Fig. 6. However, typical values of $T$ are normally set much lower than $10 \mathrm{~dB}$ since reliable QoS can be provided at lower SINR levels. Therefore, at practical values of $T$, maximum ABRP connectivity is a better user association scheme since it minimizes the required deployment factor. In other words, a HetNet with maximum ABRP connectivity requires a lower combination of optimal BS density and transmit power which minimizes the APC.

Fig. 8 shows the variation of the required $\mathcal{H}_{s, c}$ and $\mathcal{H}_{s, r}$ with their respective constraint ratios $\epsilon$ and $\kappa$ in an unbiased HetNet using maximum i-SINR and minimum BTD schemes. In general, both $\mathcal{H}_{s, c}$ and $\mathcal{H}_{s, r}$ increase with their respective constraint ratios. For minimum BTD scheme, when $T=0 \mathrm{~dB}$ and $\epsilon=\kappa, \mathcal{H}_{s, c}$ and $\mathcal{H}_{s, r}$ are equal. However, when $T<0 \mathrm{~dB}, \mathcal{H}_{s, r}>\mathcal{H}_{s, c}$ and when $T>0 \mathrm{~dB}, \mathcal{H}_{s, c}>\mathcal{H}_{s, r}$. This response is similar to the maximum ABRP case in Fig. 6 and also justifies why both constraints are required. Fig. 8 also shows that using minimum BTD scheme generally requires a lower deployment factor than maximum i-SINR scheme. 


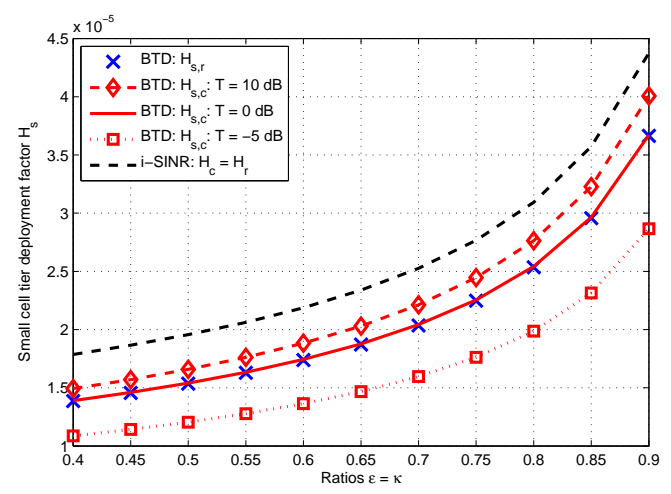

Fig. 8. Variation of $\mathcal{H}_{s, c}$ and $\mathcal{H}_{s, r}$ with ratios $\epsilon$ and $\kappa$ in the unbiased HetNet using minimum BTD and maximum i-SINR scheme $(\alpha=4)$.

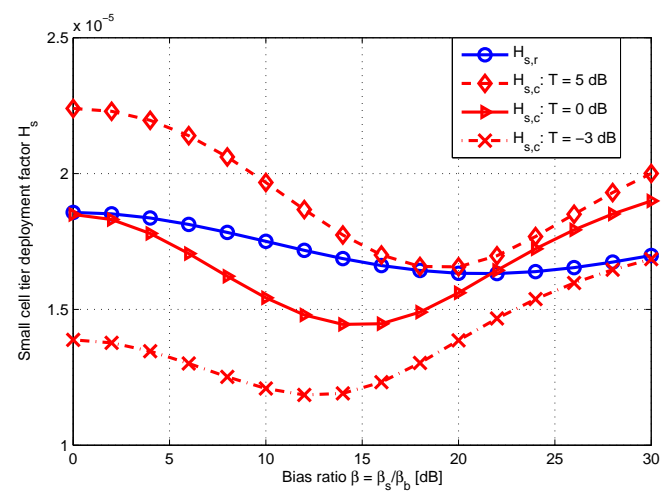

Fig. 9. Variation of $\mathcal{H}_{s}$ in a maximum ABRP-based biased HetNet (for $\mathcal{H}_{b}=2 \times 10^{-5} \mathrm{Watts} / \mathrm{m}^{2}$ and $\epsilon=\kappa=0.9$ ).

Given $\mathcal{H}_{b}$, the effect of biasing on the required small cell tier deployment factor $\mathcal{H}_{s}$ can be investigated. Fig. 9 shows the variation of $\mathcal{H}_{s, c}$ and $\mathcal{H}_{s, r}$ with the bias ratio in a HetNet using maximum ABRP connectivity, where $\epsilon=\kappa$ and $T=\{-3,0,5\} \mathrm{dB}$. Whereas $\mathcal{H}_{s, r}$ is independent of $T, \mathcal{H}_{s, c}$ is an increasing function of $T$. Hence if $T$ is sufficiently large, the overall deployment factor $\mathcal{H}_{s} \equiv \mathcal{H}_{s, c}$ over the $\beta$-range shown in Fig. 9. Conversely, if $T$ is sufficiently small, $\mathcal{H}_{s} \equiv \mathcal{H}_{s, r}$ over the same $\beta$-range. In general, $\mathcal{H}_{s, c}$ and $\mathcal{H}_{s, r}$ vary differently with the bias ratio but both show a minimum point over the $\beta$-range. Their exact minima are influenced by the value of $\mathcal{H}_{b}$ and the ratios $\epsilon$ and $\kappa$. As the bias ratio increases, both $\overline{\mathcal{P}}_{c}$ and $\overline{\mathcal{R}}_{u}$ reduce accordingly as shown in Fig. 10. This initially makes it easier to achieve the performance constraints and both $\mathcal{H}_{s, c}$ and $\mathcal{H}_{s, r}$ reduce as shown in Fig. 9. However, the small cell tier begins to dominate the macrocell tier at high bias ratios. Eventually a point is reached beyond which 


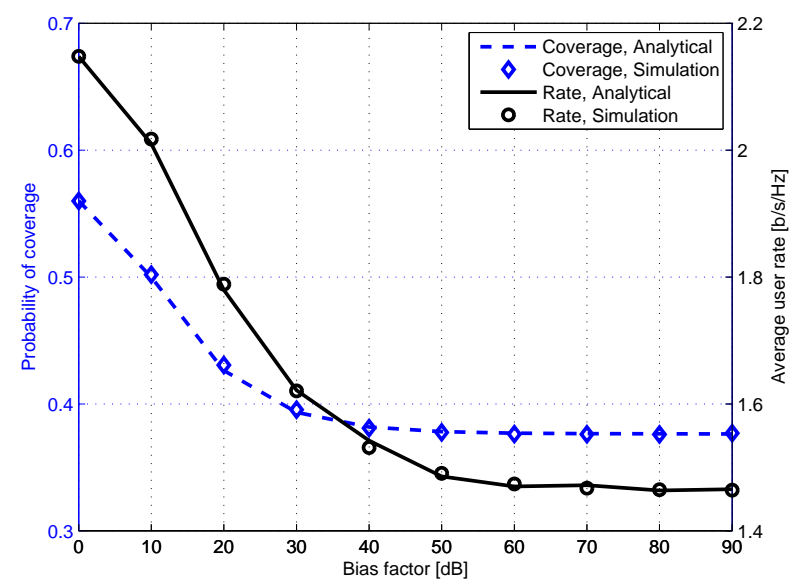

Fig. 10. $\overline{\mathcal{P}}_{c}$ and $\overline{\mathcal{R}}_{u}$ versus bias factor for a HetNet using maximum ABRP connectivity $\left(\mathcal{H}_{b}=2 \times 10^{-5} \mathrm{Watts} / \mathrm{m}^{2}\right.$ and $\left.\lambda_{s}=10^{-4} \mathrm{~m}^{-2}\right)$.

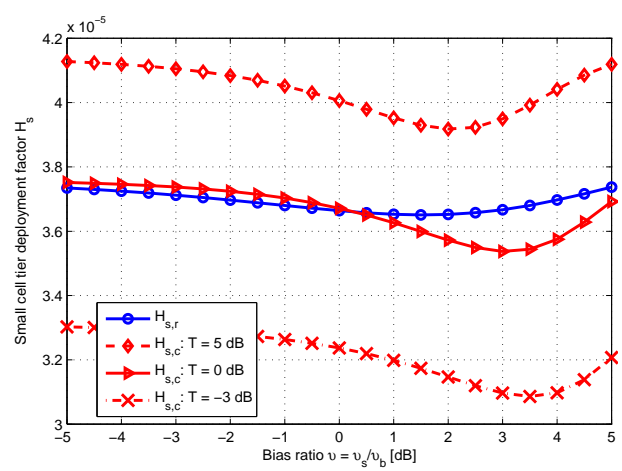

Fig. 11. Variation of $\mathcal{H}_{s}$ with bias ratio $v$ in a minimum BTD-based HetNet $\left(\mathcal{H}_{b}=10^{-6}\right.$ Watts $/ \mathrm{m}^{2}, \epsilon=\kappa=0.9$ and $\left.\alpha=4\right)$.

both $\overline{\mathcal{P}}_{c}$ and $\overline{\mathcal{R}}_{u}$ become approximately invariant with $\beta$. Since the HetNet now behaves like a homogeneous network of small BSs, it requires further densification to achieve both performance targets. This explains why both deployment factors $\mathcal{H}_{s, c}$ and $\mathcal{H}_{s, r}$ increase in this high $\beta$-regime.

For minimum BTD scheme, Fig. 11 shows the variation of $\mathcal{H}_{s, c}$ and $\mathcal{H}_{s, r}$ with bias ratio $v=v_{s} / v_{b}$. Similar to maximum ABRP scheme, $\mathcal{H}_{s, c}$ is an increasing function of $T$ but $\mathcal{H}_{s, r}$ is independent of $T$. Hence there is a range of $T$ values over which $\mathcal{H}_{s, c}$ and $\mathcal{H}_{s, r}$ are comparable, for instance at $T=0 \mathrm{~dB}$. Thus, when $T$ is sufficiently large over the considered $v$-range, $\mathcal{H}_{s} \equiv \mathcal{H}_{s, c}$ and when it is sufficiently small, $\mathcal{H}_{s} \equiv \mathcal{H}_{s, r}$. In general, both $\mathcal{H}_{s, c}$ and $\mathcal{H}_{s, r}$ are minimized at some optimal bias ratios $v_{c}^{\star}$ and $v_{r}^{\star}$ respectively. However both $v_{c}^{\star}$ and $v_{r}^{\star}$ correspond to the range $v>0 \mathrm{~dB}$ which means that user association is biased towards the macrocell tier. 


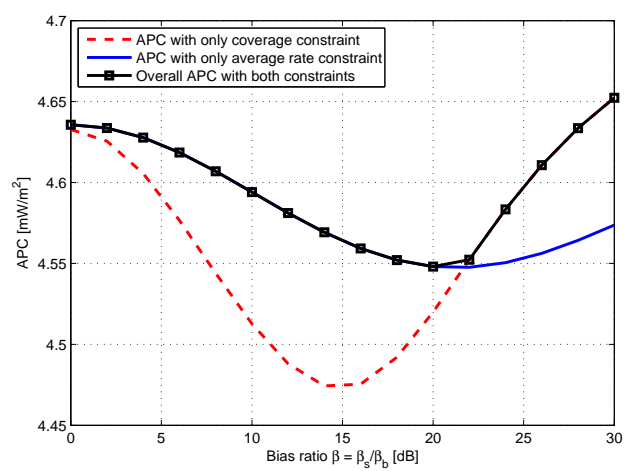

Fig. 12. Variation of APC with bias factor for maximum ABRP-based HetNet (for $\mathcal{H}_{b}=2 \times 10^{-5}$ Watts $/ \mathrm{m}^{2}, \epsilon=\kappa=0.9$ and $T=0 \mathrm{~dB})$

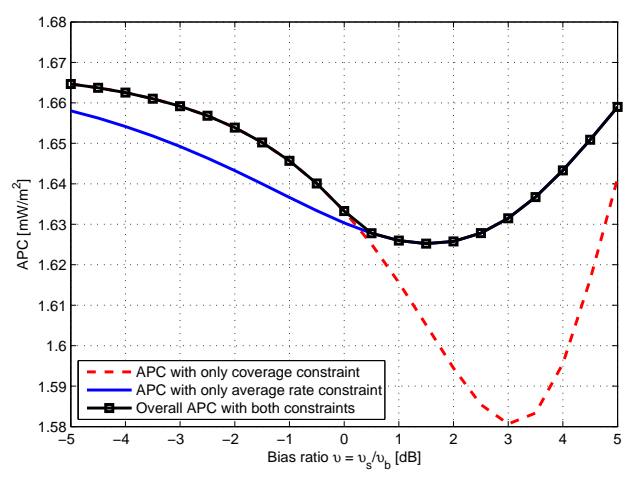

Fig. 13. Variation of APC with bias factor for minimum BTD-based HetNet $\left(\mathcal{H}_{b}=10^{-6} \mathrm{Watts} / \mathrm{m}^{2}, \epsilon=\kappa=0.9\right.$ and $\left.\alpha=4\right)$.

In the practical biasing range of $v<0 \mathrm{~dB}$, both $\mathcal{H}_{s, c}$ and $\mathcal{H}_{s, r}$ generally increase as $v$ reduces.

If $\beta^{\star}$ as the optimal bias ratio at which the overall deployment factor $\mathcal{H}_{s}=\max \left\{\mathcal{H}_{s, c}, \mathcal{H}_{s, r}\right\}$ is minimized. It follows that the APC of the HetNet is also minimized at $\beta^{\star}$. Fig. 12 shows the variation of APC with bias ratio $\beta$ in a HetNet using maximum ABRP scheme. The figure shows an optimal bias ratio $\beta^{\star}=20 \mathrm{~dB}$. Compared to the unbiased HetNet, operating the HetNet at this optimal point gives a power saving of approximately $8.4 \mathrm{~kW}$ over the simulation area. This clearly demonstrates that significant energy savings are possible if the HetNet is biased appropriately. Therefore, in addition to its load balancing potential, biasing can potentially enhance the energy performance of the HetNet by minimizing its required BS density and transmit power per tier.

For minimum BTD connectivity, the range of bias ratio is different and for this reason, its APC performance is illustrated separately in Fig. 13. The variation of APC with $v$ also gives a minimum point at which the HetNet is optimal. Fig. 13 shows that the optimal bias ratio $v^{\star}$ 
at which APC is minimized is $v^{\star}=1.5 \mathrm{~dB}$. However, this $v^{\star}$ is impractical since $v^{\star}>0 \mathrm{~dB}$ essentially means that user association is biased towards the macrocell tier. In the practical range of bias ratios (i.e. $v \leq 0 \mathrm{~dB}$ ), APC generally increases as $v$ decreases which means that the unbiased HetNet always consumes less energy than the biased HetNet. Therefore, any biasing for load balancing and other purposes has to be traded off for energy consumption. This makes maximum ABRP connectivity more desirable for biased HetNets.

\section{CONCLUSiONS}

In this paper, we developed analytical models using appropriate and simple approximations to determine energy efficient deployment configurations of unbiased HetNets in closed form. The deployment configurations vary based on user association schemes and HetNet performance constraints. Results showed that maximum ABRP connectivity scheme generally has a better energy performance than maximum instantaneous SINR connectivity scheme because it requires a lower combination of BS density and transmit power. Although biasing reduces the coverage probability and spectral efficiency of the HetNet, it is necessary in HetNets for load balancing objectives. Our results showed that for a HetNet using maximum ABRP connectivity scheme, appropriate biasing can enable the operator to make significant energy savings by reducing the BS density and transmit power required to achieve performance targets in all cellular environments. For a HetNet using minimum BTD connectivity however, biasing in the practical range deteriorates its energy performance compared to its unbiased counterpart. In addition, maximum ABRP connectivity ensures a lower HetNet deployment factor compared with maximum i-SINR connectivity. Therefore, maximum ABRP connectivity is the best association scheme from an energy consumption perspective. It can further be concluded that minimum BTD scheme is also better than maximum i-SINR scheme since it requires a lower deployment factor.

\section{APPENDIX}

\section{A. Proof of Lemma 1}

Consider that the typical user is associated to a $k$-th tier BS at a distance $r_{k}$ away. Then,

$$
\begin{aligned}
\mathcal{A}_{k} & =\mathbb{P}[m=k]=\mathbb{E}_{r_{k}}\left[\mathbb{P}\left[v_{k} r_{k}<v_{j} r_{j}, \forall j \in K, j \neq k\right]\right] \\
& =\int_{0}^{\infty} \prod_{j, j \neq k} \mathbb{P}\left[r_{j}>\frac{v_{k}}{v_{j}} r\right] f_{r_{k}}(r) \mathrm{d} r .
\end{aligned}
$$


Using the null probability of a 2-D Poisson process [14],

$$
\begin{gathered}
f_{r_{k}}(r)=\frac{\mathrm{d} \mathbb{P}\left[r_{k}>r\right]}{\mathrm{d} r}=2 \pi \lambda_{k} r e^{-\pi \lambda_{k} r^{2}}, \text { and } \\
\prod_{j, j \neq k} \mathbb{P}\left[r_{j}>\frac{v_{k}}{v_{j}} r\right]=\prod_{j, j \neq k} \mathbb{P}[\text { No } j \text {-th tier BS closer than } \\
\left.\frac{v_{k}}{v_{j}} r\right]=\prod_{j, j \neq k} e^{-\pi \lambda_{j}\left(\frac{v_{k}}{v_{j}} r\right)^{2}} .
\end{gathered}
$$

Substituting (52)-(53) into (51) and evaluating the integral gives the result in Lemma 1.

\section{B. Proof of Lemma 2}

The probability that $X_{k}>x$ is expressed as

$$
\mathbb{P}\left[X_{k}>x\right]=\mathbb{P}\left[r_{k}>x \mid m=k\right]=\frac{\mathbb{P}\left[r_{k}>x, m=k\right]}{\mathbb{P}[m=k]},
$$

where $\mathbb{P}[m=k]=\mathcal{A}_{k} . \mathbb{P}\left[r_{k}>x, m=k\right]$ is expressed as

$$
\begin{aligned}
\mathbb{P}\left[r_{k}>x, m=k\right] & =\mathbb{P}\left[r_{k}>x, v_{k} r_{k}<v_{j} r_{j}, \forall j \in K, j \neq k\right] \\
& =\int_{x}^{\infty} \prod_{j=1, j \neq k}^{K} \mathbb{P}\left[r_{j}>\frac{v_{k}}{v_{j}} r\right] f_{r_{k}}(r) \mathrm{d} r \\
& \stackrel{(a)}{=} 2 \pi \lambda_{k} \int_{x}^{\infty} r e^{-\pi \sum_{j=1}^{K} \lambda_{j}\left(\frac{v_{k}}{v_{j}}\right)^{2} r^{2}} \mathrm{~d} r,
\end{aligned}
$$

where (a) follows from (52) and (53). Therefore, substituting (18) and (55) into (54) gives

$$
\mathbb{P}\left[X_{k}>x\right]=\frac{2 \pi \lambda_{k}}{\mathcal{A}_{k}} \int_{x}^{\infty} r e^{-\pi \sum_{j=1}^{K} \lambda_{j}\left(\frac{v_{k}}{v_{j}}\right)^{2} r^{2}} \mathrm{~d} r .
$$

However, the CDF $F_{X_{k}}(x)=1-\mathbb{P}\left[X_{k}>x\right]$. Hence the PDF $f_{X_{k}}(x)=\frac{\mathrm{d} F_{X_{k}}(x)}{\mathrm{d} x}$.

\section{Proof of Theorem 1}

In this scheme, $\mathcal{L}_{I_{j}}\left(s_{c}\right)$ in (6) is expressed as

$$
\begin{aligned}
\mathcal{L}_{I_{j}}\left(s_{c}\right) & =\mathbb{E}\left[e^{-s_{c} I_{j}}\right]=\mathbb{E}_{\Phi_{j}}\left[e^{-T x^{\alpha} \widehat{P}_{j} \sum_{j \in \Phi_{j}}^{K} h_{j} r_{j}^{-\alpha}}\right] \\
& \stackrel{(a)}{=} \exp \left\{-2 \pi \lambda_{j} \int_{q_{j}}^{\infty}\left(1-\frac{1}{1+T x^{\alpha} \widehat{P}_{j} q^{-\alpha}}\right) q \mathrm{~d} q\right\} \\
& =\exp \left\{-2 \pi \lambda_{j} \int_{q_{j}}^{\infty} \frac{q}{1+\left(T x^{\alpha} \widehat{P}_{j}\right)^{-1} q^{\alpha}} \mathrm{d} q\right\},
\end{aligned}
$$


where $(a)$ follows from the probability generating functional (PGFL) of the PPP [22], [23] and the channel gain being i.i.d exponential. The limit $q_{j}$, which refers to the distance to the nearest interferer, is $q_{j}=\left(v_{k} / v_{j}\right) x$ (see (53)). Let $u=\left(T x^{\alpha} \widehat{P}_{j}\right)^{-2 / \alpha} q^{2}$ such that $q \mathrm{~d} q=\left(T x^{\alpha} \widehat{P}_{j}\right)^{2 / \alpha} \mathrm{d} u / 2$. The lower limit $u_{j}$ becomes $u_{j}=\left(T \widehat{P}_{j}\right)^{-2 / \alpha}\left(v_{k} / v_{j}\right)^{2}$. Hence,

$$
\begin{aligned}
\mathcal{L}_{I_{j}}\left(s_{c}\right) & =\exp \left\{-\pi \lambda_{j} \int_{u_{j}}^{\infty} \frac{\left(x^{\alpha} T \widehat{P}_{j}\right)^{2 / \alpha}}{1+u^{\alpha / 2}} \mathrm{~d} u\right\} \\
& =\exp \left\{-\pi \lambda_{j} \widehat{P}_{j}^{2 / \alpha} \mathcal{S}_{j} x^{2}\right\} .
\end{aligned}
$$

Combining (6), (18), (19) and (57) gives

$$
\mathcal{P}_{c, k}=\frac{2 \pi \lambda_{k}}{\mathcal{A}_{k}} \int_{0}^{\infty} e^{-\frac{T \sigma^{2}}{P_{k} L} x^{\alpha}-\pi\left\{\sum_{j=1}^{K} \lambda_{j}\left[\widehat{P}_{j}^{\frac{2}{\alpha}} \mathcal{S}_{j}+\left(\frac{v_{k}}{v_{j}}\right)^{2}\right]\right\} x^{2}} x \mathrm{~d} x .
$$

Combining (5) and (58) and substituting $z=x^{2}$ into the resulting integral gives the result.

\section{Proof of Theorem 2}

Similar to $\mathcal{L}_{I_{j}}\left(s_{c}\right)$ in $(57), \mathcal{L}_{I_{j}}\left(s_{r}\right)$ is also proved as $\mathcal{L}_{I_{j}}\left(s_{r}\right)=\exp \left\{-\pi \lambda_{j} \widehat{P}_{j}^{2 / \alpha} \mathcal{U}_{j}(t) x^{2}\right\}$. Combining $\mathcal{L}_{I_{j}}\left(s_{r}\right)$ with (5), (7), (18) and (19) and substituting $z=x^{2}$ gives the result.

\section{REFERENCES}

[1] Cisco, "Cisco Visual Networking Index: Global Mobile Data Traffic Forecast Update, 2014-2019”, White Paper, Feb. 2015.

[2] J. Zander and P. Mahonen, "Riding the Data Tsunami in the Cloud: Myths and Challenges in Future Wireless Access," IEEE Communications Magazine, Vol. 51(3), pp. 145-151, March 2013.

[3] N. Bhushan, et al., "Network Densification: The Dominant Theme for Wireless Evolution into 5G," IEEE Communications Magazine, Vol. 52(2), pp. 82-89, Feb. 2014.

[4] C. Li et al, "Throughput and Energy Efficiency Analysis of Small Cell Networks with Multi-Antenna Base Stations," IEEE Trans. on Wireless Commun., Vol.13(5), pp. 2505-2517, May 2014.

[5] Lu Lu, G.Y. Li, A. L. Swindlehurst, A. Ashikhmin, and R. Zhang, "An Overview of Massive MIMO: Benefits and Challenges," IEEE Journ. Sel. Topics in Signal Processing, Vol.8(5), pp. 742-758, Oct. 2014.

[6] H. ElSawy et al., "Two-Tier HetNets with Cognitive Femtocells: Downlink Performance Modeling and Analysis in a MultiChannel Environment," IEEE Trans. on Mob. Comp., Vol.13(3), pp. 649-663, Mar. 2014.

[7] T. Rappaport et al., "Millimetre Wave Mobile Communications for 5G Cellular: It Will Work!," IEEE Access, Vol.1, pp. 335-349, 2013.

[8] J. G. Andrews et al., "What will 5G Be", IEEE Journal on Selected Areas in Communications, Vol.32(6), pp. 1065-1082, June 2014.

[9] A. Damnjanovic, et al. "A survey on 3GPP Heterogeneous Networks," IEEE Wir. Commun. Mag., Vol.18(3), pp. 10-21, June 2011.

[10] J. G. Andrews, "Seven ways that HetNets are a Cellular Paradigm Shift," IEEE Commun. Mag., Vol.51(3), pp. 136-144, March 2013. 
[11] J. Weitzen, et al. "Large-Scale Deployment of Residential Small Cells," Proc. of the IEEE, Vol. 101(11), pp. 2367-2380, Nov. 2013.

[12] M. A. Marsan et al., "Optimal Energy Savings in Cellular Access Networks," IEEE Int. Conf. Commun. Wkshps, pp. 1-5, June 2009.

[13] Q. Ye, et al., "User Association for Load Balancing in Heterogeneous Cellular Networks", IEEE Transactions on Wireless Communications, Vol.12(6), pp. 2706-2716, June 2013.

[14] H. Jo, et al., "Heterogeneous Cellular Networks with Flexible Cell Association: A Comprehensive Downlink SINR Analysis," IEEE Trans. Wireless Commununications, Vol.11(10), pp. 3484-3495, Oct. 2012.

[15] Y. S. Soh, et al. "Energy Efficient Heterogeneous Cellular Networks", IEEE Journ. Sel. Areas in Commun., Vol.31(5), pp. 840-850, May 2013.

[16] J. Wu, et al., "Traffic-Aware Base Station Sleeping Control and Power Matching for Energy-Delay Tradeoffs in Green Cellular Networks”, IEEE Trans. Wireless Commun., Vol.12(8), pp. 4196-4209, Aug. 2013.

[17] E. Mugume and D.K.C. So, "Sleep Mode Mechanisms in Dense Small Cell Networks", IEEE Intern. Conf. Commun. (ICC), June 2015.

[18] J. Tang, D.K.C. So, E. Alsusa, and K. Hamdi, "Resource Efficiency: A New Paradigm on Energy Efficiency and Spectral Efficiency Tradeoff”, IEEE Trans. Wireless Commun., Vol.13(8), pp. 4656-4669, Aug. 2014.

[19] D. Cao, et al., "Optimal Combination of Base Station Densities for Energy-Efficient Two-Tier Heterogeneous Cellular Networks” IEEE Trans. Wireless Communications, Vol.12(9), pp. 4350-4362, Sept. 2013.

[20] J. Peng, P. Hong and K. Xue, "Energy-Aware Cellular Deployment Strategy Under Coverage Performance Constraints," IEEE Transactions on Wireless Communications, Vol.14(1), pp. 69-80, Jan 2015.

[21] M. Haenggi et al., "Stochastic Geometry and Random Graphs for the Analysis and Design of Wireless Networks," IEEE Journal on Selected Areas in Commun., Vol. 27(7), pp. 1029-1046, Sept. 2009.

[22] D. Stoyan, W. Kendall, and J. Mecke, Stochastic Geometry and its Applications, 2nd Edition, John Wiley and Sons, 1996.

[23] J.G. Andrews, F. Baccelli, and R.K. Ganti, "A Tractable Approach to Coverage and Rate in Cellular Networks", IEEE Transactions on Communications, Vol.59(11), pp. 3122 - 3134, Nov. 2011.

[24] H. S. Dhillon, et al., "Modeling and Analysis of K-Tier Downlink Heterogeneous Cellular Networks," IEEE Journal on Selected Areas in Communications, Vol.30(3), pp. 550 - 560, April 2012.

[25] H.S. Dhillon, R.K. Ganti, F. Baccelli, and J.G. Andrews, "Coverage and Ergodic Rate in $K$-Tier Downlink Heterogeneous Cellular Networks," 49th Annual Allerton Conf. on Communication, Control, and Computing, pp. 1627 - 1632, Sept. 2011.

[26] S. Singh, H. S. Dhillon, and J. G. Andrews, "Offloading in Heterogeneous Networks: Modeling, Analysis and Design Insights," IEEE Trans. on Wireless Communications, Vol.12(5), pp. 2484-2497, May 2013.

[27] A. Shojaeifard, K. A. Hamdi, E. Alsusa, D. K. C. So and J. Tang, "A Unified Model for the Design and Analysis of Spatially-Correlated Load-Aware HetNets,” IEEE Trans. on Commun., Vol.62(11), pp. 4110-4125, Nov. 2014.

[28] E. Mugume, D. K. C. So and E. Alsusa, "Energy Efficient Deployment of Dense Heterogeneous Cellular Networks," IEEE Global Communications Conference (GLOBECOM), San Diego, CA, USA, Dec. 2015, pp. 1-6.

[29] G. Auer et al., "How Much Energy is Needed to Run a Wireless Network?," IEEE Wir. Commun. Mag., Vol. 18(5), pp. $40-49,2011$.

[30] R.L. Burden and J.D. Faires, Numerical Analysis: 2.1: The Bisection Method, 9th Ed., Brooks/Cole, Cengage Learning, 2011.

[31] I.S. Gradshteyn and I.M. Ryzhik, Table of Integrals, Series, and Products, 7th Ed., Academic Press, 2007. 


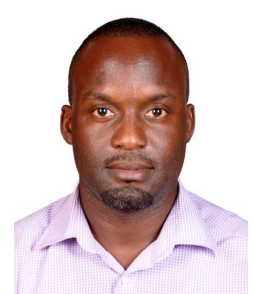

Edwin Mugume (M'17) received the BSc degree in Electrical Engineering (First Class Honours) from Makerere University, Uganda in 2007, and the MSc degree in Communication Engineering (Distinction) from the University of Manchester, United Kingdom in 2011. He completed the PhD degree in Electrical and Electronic Engineering from the University of Manchester, United Kingdom in 2016.

From 2008 to 2010, he was a radio planning and optimization engineer of mobile cellular networks at various companies in Uganda including Zain, Nokia Siemens Networks and Bharti Airtel. He also worked as a Teaching Assistant in the Department of Electrical Engineering at Makerere University. In 2012, he was an Assistant Lecturer in the Department of Electrical and Computer Engineering, Makerere University.

From 2012 to 2016, he did his PhD focusing on energy efficient deployment of future dense heterogeneous cellular networks. His research interests include green communications, dense heterogeneous networks, 5G networks, energy harvesting networks and cognitive radio.

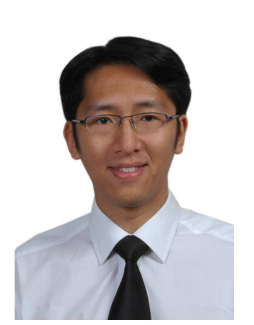

Daniel K. C. So Daniel K. C. So (S'96-M'03-SM'14) received the BEng degree in Electrical and Electronics Engineering (First Class Honor) from the University of Auckland, New Zealand in 1996, and the $\mathrm{PhD}$ degree in Electrical and Electronics Engineering from the Hong Kong University of Science and Technology (HKUST), Hong Kong in 2003.

From 1997 to 1998, he joined the Orion Systems International Limited, New Zealand, as a software engineer, and was later promoted to senior software engineer. Since 2003, he has been a lecturer and now a senior lecturer in the School of Electrical and Electronics Engineering, The University of Manchester, UK. He has also been serving as the Director of Postgraduate Taught since 2009.

His research interests include green communications, 5G networks, heterogeneous networks, cognitive radio, non-orthogonal multiple access, massive MIMO, D2D communications, cooperative MIMO schemes, multihop communication, channel equalization and estimation techniques. He is an editor of IEEE Wireless Communication Letters, and also a track co-chair for VTC Spring 2016 and 2017. 